\title{
Permutation groups of finite Morley rank
}

Borovik, Alexandre and Cherlin, Gregory 2007

MIMS EPrint: 2007.108

Manchester Institute for Mathematical Sciences

School of Mathematics

The University of Manchester

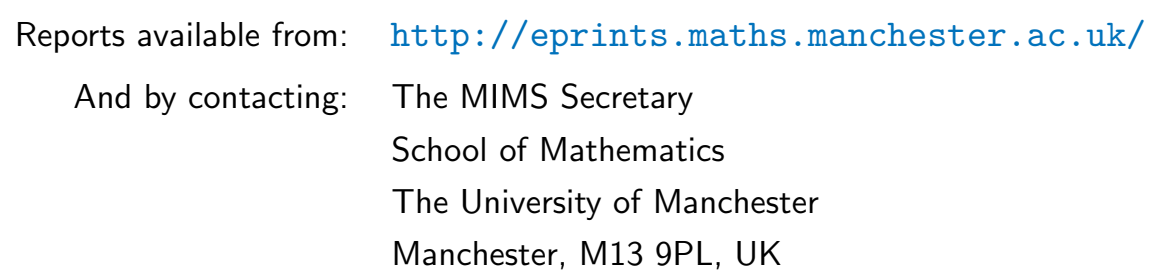

ISSN 1749-9097 


\title{
PERMUTATION GROUPS OF FINITE MORLEY RANK
}

\author{
August 2007 \\ ALEXANDRE BOROVIK \\ SCHOOL OF MATHEMATICS, \\ THE UNIVERSITY OF MANCHESTER, U.K. \\ AND \\ GREGORY CHERLIN \\ DEPARTMENT OF MATHEMATICS, \\ RUTGERS UNIVERSITY, U.S.A.
}

\section{INTRODUCTION}

Groups of finite Morley rank made their first appearance in model theory as binding groups, which are the key ingredient in Zilber's ladder theorem and in Poizat's explanation of the Picard-Vessiot theory. These are not just groups, but in fact permutation groups acting on important definable sets. When they are finite, they are connected with the model theoretic notion of algebraic closure. But the more interesting ones tend to be infinite, and connected.

Many problems in finite permutation group theory became tractable only after the classification of the finite simple groups. The theory of permutation groups of finite Morley rank is not very highly developed, and while we do not have anything like a full classification of the simple groups of finite Morley rank in hand, as a result of recent progress we do have some useful classification results as well as some useful structural information that can be obtained without going through an explicit classification. So it seems like a good time to review the situation in the theory of permutation groups of finite Morley rank and to lay out some natural problems and their possible connections with the body of research that has grown up around the classification effort.

The study of transitive permutation groups is equivalent to the study of pairs of groups $(G, H)$ with $H$ a subgroup of $G$, and accordingly one can read much of general group theory as permutation group theory, and vice versa, and, indeed, a lot of what goes on in work on classification makes a good deal of sense as permutation group theory -including even the final identification of a group as a Chevalley group, which can go via Tits' theory of buildings, or in other words by recognition of the natural permutation representations of such groups. Many special topics in permutation groups tied up with structural issues were discussed in [7, Chapter 11], with an eye toward applications. See also Part III of [15].

Second author supported by NSF Grant DMS-0100794.

Both authors thank the Newton Institute, Cambridge, for its hospitality during the Model Theory and Algebra program, where the bulk of this work was carried out, as well as CIRM for its hospitality at the September 2004 meeting on Groups, Geometry and Logic, where the seed was planted. Thanks to Altinel for continued discussions all along the way. 
The most important class of permutation groups consists of the definably primitive permutation groups, and in finite group theory one has the O'Nan-ScottAschbacher classification of these groups into various families, determined mainly by the structure of the socle and the way it meets a point stabilizer. This theorem has been adapted to the context of finite Morley rank by Macpherson and Pillay [14], and is the one really general piece of work in the area to date. We will refer to this fundamental result (or set of results) as MPOSA. Also noteworthy is the classification by Hrushovski of groups acting faithfully and definably on strongly minimal sets [17, Th. 3.27], found here as Proposition 2.4, and the study by Gropp [11] of the rank two case.

It turns out that basic notions of permutation group theory such as primitivity and multiple transitivity have more than one useful analog in the context of groups of finite Morley rank, for two reasons: (a) we are interested particularly in connected groups (and, by implication, sets of Morley degree 1); (b) we are interested in generic behavior. Of course we also impose definability constraints. So we have definable primitivity and some analogs involving connectivity, and we have generic $n$-transitivity, which is far more common than ordinary $n$-transitivity. Indeed, sharp 4-transitivity cannot occur on an infinite set [12], while $\operatorname{AGL}(V)$ acts generically sharply $(n+1)$-transitively on $V$ if $V$ has dimension $n$, with $\operatorname{PGL}(V)$ generically sharply $(n+1)$-transitive on projective space, with similar, though less extreme, statements for other classical groups acting naturally.

In our first section we will explore some of the fundamental definitions and their natural variations. After that we will focus on the following problem, which can be taken up from a number of points of view:

Problem 1. Bound the rank of a definably primitive permutation group of finite Morley rank in terms of the rank of the set on which it acts.

We will show, using soft methods, that there is some such bound. Here we combine MPOSA with some ideas that have come recently out of the classification project. There are two points to the analysis. One aims to drive the stabilizer of a sufficiently long sequence of generic and independent elements to the identity, bounding the length of the sequence. This divides into two parts: (1) getting started: first bound the possible degree of generic multiple transitivity, which is the length of time one waits before anything happens; (2) moving along: once the chain of point stabilizers begins to decrease, argue that the process runs out in bounded time. In the first stage we are not very precise in our estimates, but one may expect that very good bounds should hold in this part of the process, as generically highly transitive groups should be rare outside of known examples. This is a problem which makes sense and is interesting for groups of finite Morley rank in general, for simple algebraic groups acting definably, and even for simple algebraic groups acting algebraically. In the latter case it has been solved in characteristic 0 by Popov [19], using some results of Kimura et al. on rational representations with an open orbit.

The following result, controlling what one might reasonably call "Lie rank", plays an important role in our "soft" analysis and could also be of use in more concrete approaches. 
Lemma 3.8. Let $(G, \Omega)$ be a definably primitive permutation group of finite Morley rank, $T$ a definable divisible abelian subgroup of $G, T_{0}$ its torsion subgroup, and $O(T)$ the largest definable torsion free subgroup of $T$. Then $\operatorname{rk}(T / O(T)) \leq \operatorname{rk}(\Omega)$.

The present paper has an improvisational character. In the light of recent progress (including some directly attributable to the month devoted to this topic at the Newton Institute) it occurred to us that this could be a good time to take up the topic of permutation groups afresh. We thought that it would in particular provide an interesting setting for a review of some of the neglected but interesting areas (representation theory, cohomology) where the theory in the algebraic case offers considerable food for thought. No doubt this is the case, but that is not the paper that has emerged in this round. Rather, the rank-bounding problem described above wound up giving us a sharper but somewhat narrower focus, in which the existing theory plays a substantial role, and problems of linearization are particularly highlighted. While as a result some of our favorite problems are not represented here, we found some others, some entirely new, along with some new reasons for repeating old questions. We hope some of our readers will answer some of these questions quickly, and find better versions of some of the others. Also, we hope that the optimistic tone with which various approaches are described will not give the impression that they do not require proper proofs. The line between the proven and the unproven is certainly fuzzy here and in the long run caution is the best policy (in spite of Keynes).

We also hope that we have dealt with the foundations in a satisfactory way, or, if not, that someone will take up the matter further.

We would like to take this opportunity to thank the staff at the Newton Institute (especially the gentleman who repaired the espresso machine) and the organizers, with extra thanks to Zoé Chatzidakis.

\section{Contents}

(1) Foundations

(2) Bounds on rank: examples and a reduction

(3) The definable socle

(4) Actions of finite groups on connected groups

(5) Simple permutation groups

(6) Generic multiple transitivity: extremes

(7) Problem list

\section{Foundations}

We review some basic model theoretic and permutation group theoretic notions in the context of definable group actions, for the most part under the assumption of finite Morley rank, though it may well be worth taking the extra care necessary to work in the stable category systematically. The main notions are transitivity, primitivity, and multiple transitivity and a number of related variants, along with definability, genericity, and connectivity. Most of this is already present in one form or another in [14] but we think it is still worth while to consider the foundations at leisure, and separately from more technical matters. Indeed, we have the feeling that there is still something to be done here, at least at the level of collecting illuminating examples. 
The term "permutation group" is generally taken here to refer to a group equipped with a faithful action. But in dealing with intransitive actions, we may consider the restriction of the group to an individual orbit, and there may be a kernel in this case. Sometimes this actually matters. We will be casual about this below; strictly speaking one should insert the term "faithful" almost everywhere below, taking pains to omit it occasionally.

1.1. Transitivity, genericity, connectivity. We work in the definable category, that is with groups and definable actions. We write actions on the right ( $\alpha$ " $\left.{ }^{\prime \prime}\right)$. The following is completely elementary but fundamental.

Lemma 1.1. Let $(G, \Omega)$ be a transitive permutation group, and $G_{\alpha}$ a point stabilizer (with $\alpha \in \Omega$ ). Then the action of $G$ on $\Omega$ is equivalent to the action of $G$ on the coset space $G_{\alpha} \backslash G$. In particular the action is interpretable in $G$ if and only if $\Omega$ and $G_{\alpha}$ are interpretable in $G$, in which case the two actions are definably equivalent.

One often treats permutation groups $(G, \Omega)$ as structures with underlying set $\Omega$ and some inherited relations. The most satisfying choice of relations for our present purposes, where the group $G$ is of interest in its own right, are those definable without parameters in the structure $(G, \Omega)$.

Lemma 1.2. Let $(G, \Omega)$ be a transitive permutation group and view $\Omega$ as a structure equipped with all relations 0 -definable in the two-sorted structure $(G, \Omega)$, equipped with the action. View $G$ as a structure equipped with its group operation and a distinguished subgroup $G_{\alpha}$ for some $\alpha \in \Omega$. If the structure on $G$ is stable then $G$ is interpretable in $\Omega$. In particular, if $G$ is stable and $\Omega$ has finite Morley rank, then $G$ has finite Morley rank.

Proof. The point stabilizers form a uniformly definable family of subgroups. If $G$ is stable it follows that arbitrary intersections of point stabilizers are in fact point stabilizers of finite sets. In particular the identity subgroup is the stabilizer of some finite subset $A \subseteq \Omega$. Hence the map $g \mapsto A^{g}$ is $1-1$. Therefore the elements of $G$ and the group structure on $G$ are encoded in $\Omega$.

Problem 2. If $(G, \Omega)$ is a permutation group and $\Omega$ is stable in the induced language, does it follow that $G$ is stable? Does this hold at least when $\Omega$ has finite Morley rank?

Note that in the present paper we will treat the structure $(G, \Omega)$ as given, and not just the group $G$. So in that context the action of interest is always definable, and whether it is definable in some previously given language on $G$ is a question that rarely arises for us here, though when $G$ is, for example, an algebraic group, it may be an important issue.

Genericity is a fundamental notion in groups, and passes to transitive permutation groups.

Lemma 1.3. Let $(G, \Omega)$ be a stable transitive permutation group, $\alpha \in \Omega$, and $X$ a definable subset of $\Omega$. Then the following conditions are equivalent.

(1) $\left\{g \in G: \alpha^{g} \in X\right\}$ is generic in $G$

(2) Finitely many $G$-translates of $X$ cover $\Omega$.

If $\Omega$ has finite Morley rank then an equivalent condition is

(3) $\operatorname{rk}(X)=\operatorname{rk}(\Omega)$ 
Such a set is called generic in $\Omega$.

We note that after identifying $\Omega$ with $G_{\alpha} \backslash G$, the set defined in point (1) is $\bigcup X$. We now look at connected components.

Lemma 1.4. Let $(G, \Omega)$ be a transitive permutation group of finite Morley rank. Let $\Omega_{0}$ be an orbit for the connected component $G^{\circ}$, Then $\operatorname{rk}\left(\Omega_{0}\right)=\operatorname{rk}(\Omega), \operatorname{deg}\left(\Omega_{0}\right)=1$, and the orbits of $G_{0}$ are conjugate under the action of $G$.

Proof. Since $G^{\circ} \triangleleft G$, the orbits of $G^{\circ}$ are conjugated by $G$, and as the action of $G$ is transitive it follows that these orbits are conjugate. As there are finitely many such orbits they have the same rank as $\Omega$. Finally, there is a definable bijection between $\Omega_{0}$ and a coset space for $G^{\circ}$, so the Morley degree of $\Omega_{0}$ is 1 .

We note that the setwise stabilizer $G_{0}=G_{\left\{\Omega_{0}\right\}}$ of $\Omega_{0}$ may be larger than $G^{\circ}$ and that the former is really the group induced "by $G$ " on $\Omega_{0}$.

It is useful to extend the notion of genericity to permutation groups which are not necessarily transitive, at least in the context of groups of finite Morley rank. This we do using the rank directly.

Definition 1.5. Let $X$ be a definable set in a structure of finite Morley rank. A definable subset $Y$ of $X$ is strongly generic in $X$ if $\operatorname{rk}(Y \backslash X)<\operatorname{rk}(X)$, and weakly generic in $X$ if $\operatorname{rk}(Y)=\operatorname{rk}(X)$. When $X$ has Morley degree 1, the two notions are equivalent, and are referred to as genericity. Otherwise, it is prudent to specify which version is meant.

Lemma 1.6. Let $(G, \Omega)$ be a transitive permutation group of finite Morley rank and $g$ an element of $G$ which fixes a strongly generic subset of $\Omega$ pointwise. Then $g=1$.

Proof. Let $H=\{g \in G: g$ fixes a strongly generic subset of $\Omega$ pointwise $\}$. Then $H$ is a definable normal subgroup of $G$.

Take $h \in H^{\circ}$ generic, and $\alpha \in \Omega$ generic over $h$. Then $h$ and $\alpha$ are independent, so $\alpha$ is fixed by a generic subset of $H^{\circ}$, and hence by $H^{\circ}$. As $H^{\circ}$ is normal in $G$ and $G$ acts transitively, the group $H^{\circ}$ acts trivially on $\Omega$. That is, $H^{\circ}=1$ and $H$ is finite.

Let $X$ be the fixed point set for $H$ in $\Omega$. As $H$ is finite, the set $X$ is generic, hence nonempty. Since $X$ is also $G$-invariant, we have $X=\Omega$ and thus $H=1$.

Definition 1.7. Let $(G, \Omega)$ be a permutation group.

(1) $G$ is generically transitive on $\Omega$ if $G$ has a strongly generic orbit.

(2) $G$ is generically $n$-transitive on $\Omega$ if the induced action on $\Omega^{n}$ is generically transitive.

(3) $G$ is generically sharply $n$-transitive on $\Omega$ if the induced action on $\Omega^{n}$ has a strongly generic orbit on which $G$ acts regularly.

We will not actually take up the generically sharply transitive case per se, but it is worth mentioning as the subject of [11], particularly since such groups certainly exist.

Example 1. The natural representation of $\mathrm{AGL}(n)$ affords a generically sharply $(n+1)$-transitive action, and the natural projective representation of $\mathrm{PGL}(n)$ affords a generically sharply $(n+1)$-transitive action. 
Problem 3. Find all the generically sharply $n$-transitive actions of algebraic groups over algebraically closed fields, for $n \geq 2$.

Note that by a theorem of Hall [12] there cannot be a sharply $n$-transitive permutation group on an infinite set for $n \geq 4$, which stands in sharp contrast to the above.

We would suggest that it is reasonable and interesting, though certainly challenging, to aim eventually at an identification of all the generically highly transitive groups of finite Morley rank.

While there is no close connection in general between generic $n$-transitivity and $n$-transitivity, it is reasonable to work with transitive actions throughout (unless the set $\Omega$ carries some useful structure supported on several orbits, as is the case in most natural representations). For $n=1$ one just restricts to the strongly generic orbit (possibly picking up a kernel). For $n>1$ one makes use of the following.

Lemma 1.8. Let $(G, \Omega)$ be a generically $n$-transitive permutation group of finite Morley rank.

(1) $(G, \Omega)$ is generically $m$-transitive for $m \leq n$.

(2) If $X$ is the strongly generic orbit for $G$, then $G$ is generically $n$-transitive on $X$

(3) If $n>1$ then $\Omega$ has Morley degree 1 .

Proof. The first two points are clear. For the last point, we may suppose that $G$ is transitive on $\Omega$. If $\Omega$ has Morley degree greater than 1 , then $\Omega$ contains distinct $G^{\circ}$-orbits $\Omega_{0}$ and $\Omega_{1}$. But then no element of $\Omega_{0} \times \Omega_{0}$ is conjugate to any element of $\Omega_{0} \times \Omega_{1}$ under the action of $G$, and we have a contradiction.

The last argument is a very weak analog, but the best we have, for the statement that a doubly transitive group is primitive.

Lemma 1.9. Let $(G, \Omega)$ be a generically $n$-transitive permutation group, and let $\Omega_{0}$ be a $G^{\circ}$-orbit. Then $\left(G^{\circ}, \Omega_{0}\right)$ is generically $n$-transitive.

Proof. If $n=1$ then this is clear. If $n>1$ then $\Omega$ has Morley degree 1 , so $\Omega^{n}$ also has Morley degree 1. If $O$ is the strongly generic orbit for $G$ in $\Omega^{n}$, and $O_{0}$ is a $G^{\circ}$-orbit inside $O$, then $\operatorname{rk}\left(O_{0}\right)=\operatorname{rk}(O)=\operatorname{rk}\left(\Omega^{n}\right)$ and as the Morley degree is 1 it follows that $O_{0}$ is strongly generic in $\Omega^{n}$.

1.2. Notions of Primitivity. Let us propose a number of notions of primitivity.

Definition 1.10. Let $(G, \Omega)$ be a permutation group.

(1) The action is primitive if there is no nontrivial G-invariant equivalence relation.

(2) The action is definably primitive if there is no nontrivial definable $G$ invariant equivalence relation.

(3) The action is virtually definably primitive if any G-invariant definable equivalence relation has either finite classes or finitely many classes.

We are not much interested in primitivity per se, as this is too not natural in the definable category. On the other hand it happens to be the case that almost all definably primitive permutation groups are primitive ([14], see Lemma 1.17).

It is less clear which of the definable versions of primitivity is to be preferred, and we have a third possibility to offer in a moment (this in turn raises the question 
as to what the O'Nan-Scott-Aschbacher Theorem should be about in our category). Certainly one desirable criterion is that any permutation representation that may interest us should have a nontrivial primitive quotient. These will be definable, and frequently of Morley degree 1 (or, indeed, with the acting group $G$ connected).

We intend to lay out the relationships among these notions in detail. First we give their translations into "internal" group theoretic terms. For this we will need to restrict attention to transitive group actions, so we begin with this point, which is completely straightforward.

Lemma 1.11. Let $(G, \Omega)$ be a permutation group.

(1) If the action is definably primitive, then it is transitive.

(2) If the action is virtually definably primitive then either it has finitely many orbits, and is virtually definably primitive on each orbit, or else it has finite orbits, and the set of orbits carries no definable equivalence relation with infinitely many infinite classes.

Proof. Possibly the context for the second point needs to be elucidated. The notion of definability is relative to the structure $(G, \Omega)$ containing the action and whatever additional structure it may carry (on $G$, on $\Omega$, or on both together). We consider the equivalence relation given by the orbits themselves, which is definable in this context. If the orbits are finite then this is not very interesting, but it may meet our definition.

Remark 1.12. Let $(G, \Omega)$ be a stable permutation group with all orbits finite. Then $G$ is finite, since the kernel (which is assumed trivial) is the stabilizer of a finite set, and therefore $G$ acts faithfully on a finite union of orbits.

This remark is actually of some use, when one comes across permutation groups carrying a definable invariant equivalence relation with finite classes. The lemma states that the kernel of the action on the set of classes is finite.

It should now be reasonably clear that nothing of any significance would be lost by including transitivity in the definition of virtual definable primitivity.

Lemma 1.13. Let $(G, \Omega)$ be a transitive permutation group, $\alpha \in \Omega$ fixed, and $G_{\alpha}$ the point stabilizer.

(1) The action is primitive if and only if $G_{\alpha}$ is a maximal proper subgroup of $G$.

(2) The action is definably primitive if and only if $G_{\alpha}$ is a maximal definable subgroup of $G$.

(3) The action is virtually definably primitive if and only if for any definable subgroup $H$ of $G$ containing $G_{\alpha}$, either $\left[H: G_{\alpha}\right]$ or $[G: H]$ is finite.

Proof. The $G$-invariant equivalence relations on $\Omega$ are classified by the subgroups of $G$ containing $G_{\alpha}$. More explicitly, if $C$ is a set containing $\alpha$, and $G_{\alpha: C}=\{g \in$ $\left.G: \alpha^{g} \in C\right\}$, then the following are equivalent

(1) $G_{\alpha: C}$ is a subgroup of $G$;

(2) $G_{\alpha: C}$ is the setwise stabilizer $G_{\{C\}}$ of $C$ in $G$;

(3) $\left\{C^{g}: g \in G\right\}$ is a partition of $\Omega$.

Furthermore, when this holds we have $|C|=\left[G_{\{C\}}: G_{\alpha}\right]$ and $\left|\left\{C^{g}: g \in G\right\}\right|=[G$ : $\left.G_{\{C\}}\right]$. 
There is a rule of thumb that says one is always interested in the "connected" versions of classical notions. So we propose a notion of c-primitivity intended to be the connected version of primitivity, in two variants.

Definition 1.14. Let $(G, \Omega)$ be a transitive permutation group of finite Morley rank, $\alpha \in \Omega$, and $G_{\alpha}$ the point stabilizer.

(1) The action is c-primitive if $G_{\alpha}$ is a maximal proper definable connected subgroup of $G^{\circ}$.

(2) The action is virtually c-primitive if $G_{\alpha}{ }^{\circ}$ is a maximal proper definable connected subgroup of $G^{\circ}$.

One may wish to translate this back into permutation group theoretic terms. Here one needs the notion of a finite cover $(G, \hat{\Omega})$ of a permutation group $(G, \Omega)$, which is given by a surjective $G$-invariant map $\pi: \hat{\Omega} \rightarrow \Omega$ with finite fibers (really the $\operatorname{map}(1, \pi):(G, \hat{\Omega}) \rightarrow(G, \hat{\Omega})$ is the morphism $)$.

Lemma 1.15. Let $(G, \Omega)$ be a transitive permutation group of finite Morley rank.

(1) The action is virtually c-primitive if and only if every definable finite cover is virtually definably primitive.

(2) The action is c-primitive if and only if every finite cover is an isomorphism, and the action is virtually definably primitive.

Proof. Only the first point requires unwinding, and this is simply a matter of working out the content of the cumbersome criterion given, which can be read as follows: for every definable subgroup $G_{0}$ of $G_{\alpha}$ of finite index, and for every definable subgroup $H$ of $G$ containing $G_{0}$, either $\left[H: G_{0}\right]<\infty$ or $[G: H]<\infty$. This can be decoded further to: either $H^{\circ}=G_{\alpha}{ }^{\circ}$ or $H^{\circ}=G^{\circ}$ - at which point we have virtual $c$-primitivity.

One of the goals of permutation group theory is to provide a convenient language for saying useful things about maximal subgroups, and in a finite Morley rank context one thing that one could reasonably ask of the parallel theory is a useful way of looking at maximal connected subgroups. But this is not really the point of view that has been taken to date.

We now take note of reductions to Morley degree 1.

Lemma 1.16. Let $(G, \Omega)$ be a transitive permutation group of finite Morley rank, and $\Omega_{0}$ an orbit for $G^{\circ}$ in $\Omega$. Let $G_{0}=G_{\left\{\Omega_{0}\right\}}$.

(1) If $(G, \Omega)$ is definably primitive then $\Omega$ has Morley degree 1 and $\Omega_{0}=\Omega$, $G_{0}=G$.

(2) If $(G, \Omega)$ is virtually definably primitive then any $G_{0}$-invariant definable relation on $\Omega_{0}$ has finite classes.

(3) If $(G, \Omega)$ is c-primitive or virtually c-primitive, then $\left(G_{0}, \Omega_{0}\right)$ has the same property.

On the other hand, this does not mean that we can reduce the group itself to its connected component.

Example 2. Let $(H, X)$ be a definably primitive permutation group of finite Morley rank with $H$ connected. Let $K$ be a finite permutation group acting transitively on a set $I$. Then the wreath product $H \backslash K$ acting on $X^{I}$ is a definably primitive permutation group of finite Morley rank whose connected component $H^{I}$ leaves invariant 
the equivalence relations $E_{i}(i \in I)$ defined by

$$
E_{i}(a, b) \Longleftrightarrow a_{i}=b_{i}
$$

Thus $\left(H^{I}, X^{I}\right)$ is not even virtually definably primitive.

One may still find it profitable in practice to pay particular attention to connected permutation groups, but there is no general reduction of the full theory to that case, and this leads to practical difficulties in situations where one would like to make inductive arguments.

The foregoing example is typical in the sense that the class of definably primitive groups is much richer than the set of connected definably primitive groups precisely because the finite sections can play such an important role. The general MPOSA classification includes several cases that cannot arise as actions of connected groups.

\subsection{Primitivity vs. Definable Primitivity.}

Example 3. A definably primitive action which is not primitive.

Take a large torsion free divisible abelian group in its natural language (or as $a \mathbb{Q}$-vector space) and consider the regular action. This is definably primitive and c-primitive but not primitive.

One may be a bit suspicious of this example since the point stabilizer is trivial, and with good reason. If a regular action is definably primitive then the group $G$ contains no nontrivial proper definable subgroups and hence is abelian and (if infinite) torsion free. On the other hand according to [14, Prop. 2.7] a definably primitive group is primitive unless the point stabilizer is finite. We elaborate slightly on this point.

Lemma 1.17 ([14]). Let $(G, \Omega)$ be a permutation group of finite Morley rank which is definably primitive but not primitive, and let $G_{\alpha}$ be a point stabilizer. Then $G_{\alpha}$ is finite, and if $G_{\alpha}>1$ then $G^{\circ}$ is either abelian or quasisimple.

Proof. Suppose that $G_{\alpha}<H<G$. Let $H_{0}$ be the normal closure in $H$ of $G_{\alpha}{ }^{\circ}$. Then by a lemma of Zilber $H_{0}$ is definable and connected. Since $G_{\alpha} \leq H_{0} G_{\alpha} \leq H$ it follows by definable primitivity that $H_{0}=G_{\alpha}{ }^{\circ}$, that is $G_{\alpha}{ }^{\circ} \triangleleft H$. As $G_{\alpha}<$ $N\left(G_{\alpha}{ }^{\circ}\right) \leq G$ it follows that $G_{\alpha}{ }^{\circ} \triangleleft G$. Now $G$ acts transitively on $\Omega$ so it follows that $G_{\alpha}{ }^{\circ}$ acts trivially on $\Omega$, hence $G_{\alpha}{ }^{\circ}=1$ and $G_{\alpha}$ is finite.

Now if $K$ is a definable connected normal subgroup of $G$ then $K G_{\alpha}=G$ and thus $K=G^{\circ}$. So if $G^{\circ}$ is nonabelian then $G^{\circ}$ is quasisimple, that is perfect with finite center and simple factor group.

This result has a converse: if the point stabilizer is finite it cannot be maximal, at least if we pass to an uncountable model.

Examples of such groups with $G^{\circ}$ abelian coming from irreducible representations of finite groups are mentioned in [14]. Another type of example is the following (responding to a question raised in the last paragraph of $\S 2$ of [14]).

Example 4. Let $G=\mathrm{PSL}_{2}(\mathbb{C})$ and consider the action on cosets of $H=\operatorname{Alt}(5)$. Then $H$ is maximal among Zariski-closed proper subgroups, but not among (e.g., countable) proper subgroups. So in the algebraic category this provides a definably primitive but not primitive action.

It can be shown with some additional effort that this action will remain definably primitive in any enrichment of the language for which the group has finite Morley rank. 
1.4. Definably Primitive Quotients. One would like to think that the general permutation group of finite Morley rank can be analyzed in terms of transitive and even primitive constituents. The reduction to the transitive case already involves very substantial complications. Even if the action is generically transitive, the action on a generic orbit may have nontrivial kernel, and there may be infinitely many nongeneric orbits.

As far as primitivity is concerned, one would like to find a nontrivial definably primitive quotient of any transitive permutation group of finite Morley rank, but even this is too much to ask.

Example 5. Let $G$ be a Chevalley group over an algebraically closed field of positive characteristic, and let $G(q)$ be the subgroup of $\mathbb{F}_{q}$-rational points. Typically the proper Zariski closed subgroups of $G$ containing $G(q)$ are the $G\left(q^{\prime}\right)$ for $q^{\prime}$ a power of $q$. Thus taking $G$ with its structure as an algebraic group, any proper definable subgroup containing $G(q)$ is finite, and thus if we consider the action of $G$ on $G(q) \backslash G$, this has no definable and definably primitive quotient.

On the other hand, this example is again fairly typical.

Lemma 1.18. Let $(G, \Omega)$ be a transitive permutation group of finite Morley rank. Then the following hold.

(1) There is a nontrivial definable quotient of $(G, \Omega)$ which is virtually definably primitive.

(2) If $(G, \Omega)$ is virtually definably primitive then either $(G, \Omega)$ is a finite cover of a definably primitive permutation group, or the point stabilizer is finite.

Proof. Let $G_{\alpha}$ be a point stabilizer.

The first point is immediate: extend $G_{\alpha}$ to a proper definable subgroup of maximal rank.

For the second point, if $N\left(G_{\alpha}{ }^{\circ}\right)<G$ then $\left[N\left(G_{\alpha}{ }^{\circ}\right): G_{\alpha}\right]<\infty$ by virtual definable primitivity, and we look at $N\left(G_{\alpha}{ }^{\circ}\right) \backslash G$. On the other hand, if $G_{\alpha}{ }^{\circ} \triangleleft G$ then by transitivity $G_{\alpha}{ }^{\circ}$ acts trivially, $G_{\alpha}{ }^{\circ}=1$.

The most useful instance of this is the following.

Corollary 1.19. Let $(G, \Omega)$ be a transitive permutation group of finite Morley rank with $G$ connected simple. Then either $(G, \Omega)$ has a nontrivial definably primitive quotient, or the point stabilizer is finite.

Proof. We apply both parts of the previous corollary, getting a nontrivial quotient of $(G, \Omega)$ satisfying one of our two conclusions. Note however that if the point stabilizer in the quotient is finite then it was finite in $(G, \Omega)$ : as $G$ is simple, there is no kernel in this action.

Problem 4. Suppose that $(G, \Omega)$ is a virtually definably primitive permutation group of finite Morley rank with which is not a finite cover of a definably primitive permutation group. Show that $G$ is a Chevalley group of positive characteristic, and the point stabilizer is contained in $G\left(\mathbb{F}_{q}\right)$ for some finite field $\mathbb{F}_{q}$.

As we have already noticed, $c$-primitivity is just virtual definable primitivity together with the condition that the point stabilizer be connected. On the other hand, in general virtual definable primitivity has little to do with $c$-primitivity, with examples again afforded by actions with finite point stabilizers. 
1.5. Generic $n$-Transitivity Revisited. We have the natural inductive principle: if $(G, \Omega)$ is a generically $n$-transitive permutation group of finite Morley rank, and $G_{\alpha}$ a point stabilizer with $\alpha$ in the generic orbit, then $G_{\alpha}$ acts generically $(n-1)$ transitively on $\Omega$.

However, one might also expect generically highly transitive actions to be definably primitive, and this fails badly.

Example 6. Let $\left(H_{i}, X_{i}\right)(i=1,2)$ be generically $n$-transitive permutation groups of finite Morley rank. Then $\left(H_{1} \times H_{2}, X_{1} \times X_{2}\right)$ is generically n-transitive.

One may ask whether there is, nonetheless, a general theory of generically $n$ transitive groups. There is an initial reduction to the primitive case. Recall that generic $n$-transitivity passes to connected components.

Lemma 1.20. Let $(G, \Omega)$ be a transitive and generically $n$-transitive group of finite Morley rank with $G$ connected. Then the following hold.

(1) $\operatorname{rk}\left(G_{\alpha}\right) \geq(n-1) \operatorname{rk}(\Omega)$ for $\alpha \in \Omega$.

(2) Any definable quotient of $(G, \Omega)$ is generically n-transitive.

(3) If $n>1$ then there is an infinite, definable, definably primitive quotient $(\bar{G}, \bar{\Omega})$

Proof. The first two points are immediate. For the last point, since $G$ is connected, "infinite" is the same as "nontrivial".

Now Lemma 1.18 tells us that we have an infinite definable and virtually definably primitive quotient $(\bar{G}, \bar{\Omega})$ and that this is either a finite cover of a definably primitive permutation group, or has a finite point stabilizer. But $\operatorname{rk}\left(\bar{G}_{\bar{\alpha}}\right)>0$ so the latter possibility is excluded.

We now have two good reasons for restricting our attention to the primitive case: (1) in the present article we need to deal with generically $n$-transitive groups in order to analyze primitive ones; (2) it seems that results on the primitive case may bear strongly on the general case, in view of the foregoing.

Problem 5. Is there an O'Nan-Scott-Aschbacher analysis of generically 2-transitive groups which are not necessarily definably primitive? Are all such groups essentially products of generically $n$-transitive primitive groups (or generically $n^{\prime}$-transitive groups, with $n^{\prime}$ not much smaller than $\left.n\right)$ ?

This concludes our review of the fundamental definitions. The central notion of [14] was definable primitivity, and this review suggests that one might on occasion prefer to broaden the notion a little, but that the impact of this would be marginal.

\section{BOUNDS ON RANK}

We will organize our discussion around the following result and problem.

Theorem 1. There is a function $\rho: \mathbb{N} \rightarrow \mathbb{N}$ such that the following holds. For any virtually definably primitive permutation group $(G, \Omega)$ of finite Morley rank we have

$$
\operatorname{rk}(G) \leq \rho(\operatorname{rk}(\Omega))
$$

Problem 6. Find good bounds on $\rho$, where $\rho(r)$ is the maximum rank of a virtually definably primitive permutation group $(G, \Omega)$ of finite Morley rank, with $\operatorname{rk}(\Omega)=r$. 
Notice the following corollary.

Corollary 2.1. There is a function $\tau: \mathbb{N} \rightarrow \mathbb{N}$ such that the following holds. For any virtually definably primitive permutation group $(G, \Omega)$ of finite Morley rank which is generically t-transitive,

$$
t \leq \tau(\operatorname{rk}(\Omega))
$$

Problem 7. Find good bounds on $\tau$, where $\tau(r)$ is the maximum degree of generic transitivity associated to a virtually definably primitive permutation group $(G, \Omega)$ of finite Morley rank, with $\operatorname{rk}(\Omega)=r$.

We particularly like this last problem, because we have as yet no decent bounds on $\tau$ and we imagine there should be very good ones. The gap between $\tau$ and $\rho$ is not so large, as we shall show.

Proposition 2.2. For any primitive permutation group $(G, \Omega)$ of finite Morley rank with arbitrary $r=\operatorname{rk}(\Omega)$ we have

$$
r \tau(r) \leq \rho(r) \leq r \tau(r)+\left(\begin{array}{l}
r \\
2
\end{array}\right)
$$

The proof of this Proposition will be given shortly.

Lemma 2.3. We may take $\Omega$ to have Morley degree 1, and we may replace "virtually definably primitive" by "definably primitive," in the definitions of $\rho$ and $\tau$, without altering the values.

Proof. We have seen in Lemma 1.16 that we may restrict attention to Morley degree 1 actions, with all $G$-invariant relations finite. Furthermore we know that each relevant permutation group $(G, \Omega)$ is either a finite cover of a definably primitive one, or has finite point stabilizer. It suffices to observe now that neither the rank nor the degree of generic transitivity can be maximized in the presence of a finite point stabilizer.

2.1. Examples. Our first order of business is to justify our primitivity hypotheses. After all, any permutation group on a set of rank 0 has rank 0 , which is a very good bound. There is also an excellent bound for the rank of a stable group acting transitively on a rank 1 set, due to Hrushovski.

Proposition 2.4. Let $(G, A)$ be a stable permutation group with A strongly minimal. Then the Morley rank of $G$ is at most 3 , and either $G^{\circ}$ is abelian and regular on $A$, or $G$ is isomorphic with a Zariski closed subgroup of $\mathrm{PSL}_{2}(K)$ for some algebraically closed and strongly minimal field $K$ interpretable in $G$.

In particular, if the rank of $G$ is $n \leq 3$ then the action of $G^{\circ}$ is sharply $n$-transitive on $A$.

But above rank 1 things become more complicated. Examples of the following type are given in [11], and show that nontransitive permutation groups are not so easily reduced to transitive ones.

Example 7. Let $V$ be a vector space over an algebraically closed field $K, L$ a 1 dimensional vector space over $K, E_{V}=\operatorname{End}(V), \lambda: V \rightarrow L$ a nonzero linear map, and $f: L \rightarrow V$ a map from the line $L$ into a space curve in $V$ not contained in a 
proper subspace of $V$. We associate with these data the permutation group $\left(E_{V}, L^{2}\right)$ with $E_{V}$ viewed as an additive group and with action

$$
A .(x, y)=(x, y+\lambda(A . f(x)))
$$

(using left-handed notation). Evidently this is faithful, and $L^{2}$ has rank two.

The orbits are the rank one sets $L_{x}=\{x\} \times L$. On $L_{x}$, if $v=f(x)$ then the kernel is

$$
\{A: A . v \in \operatorname{ker} \lambda\}
$$

giving a parametrized family of subspaces of $\operatorname{End}(V)$.

Even in the transitive case there are somewhat similar examples.

Example 8. Let $G$ be an algebraic group with a rational linear representation on the vector space $V$ and let $W$ be a subspace of $V$ which does not contain any nontrivial $G$-invariant subspace of $V$. Let $\hat{G}=V \rtimes G$ and consider the transitive permutation group

$$
(\hat{G}, W \backslash \hat{G})
$$

This has rank $\operatorname{rk}(G)+\operatorname{rk}(V / W)$, and is not definably primitive since $W<V$. On the other hand it is faithful by the choice of $W$.

We want $\operatorname{rk}(\hat{G})$ unbounded and $\operatorname{rk}(W \backslash \hat{G})$ bounded, for which we take $G$ fixed and $\operatorname{dim}(V / W)$ bounded. For example $G$ could be simple, $V$ irreducible, and $W$ a hyperplane. Or $G$ could be a torus acting so $V$ so that all weight spaces are 1-dimensional, and $W$ could be a hyperplane avoiding all weight spaces.

In particular $G$ could be a 1-dimensional torus and then $W \backslash \hat{G}$ would be a Morley rank two representation of a group of arbitrarily large rank.

Here a nontrivial invariant equivalence relation is given by the orbits of the normal subgroup $V$, the quotient has kernel $V$, and the induced action is the regular action of $G$ on itself. On the other hand the stabilizer of a single equivalence class will be the group $V$, with kernel a conjugate of $W$, and this portion of the action resembles the previous example.

These examples are not particularly outlandish, but they do suggest that it may be difficult to get even the coarsest degree of control over imprimitive representations.

On the other hand, we will eventually get a bound on the rank of a simple group of finite Morley rank acting definably on a set of specified rank, and from this we will get our general bound in the primitive case.

2.2. Reduction to generic multiple transitivity. We take up the proof of Proposition 2.2. The idea is that if a point stabilizer does not act generically transitively, then the ranks of the orbits of successive point stabilizers (taken along a sequence of independent generic points of $\Omega$ ) decrease steadily. One difficulty that immediately comes to mind, in view of the last example, is that we have no assurance that we can usefully pass to simple constituents of the induced permutation groups along the way. So we need to apply the initial primitivity hypothesis for the full group at each step.

On the other hand we will see later that by making use of MPOSA one can sometimes usefully recover primitivity for such constituents. So in all probability the very soft argument given here can be usefully refined, and the bounds sharpened, using both MPOSA and the structure theory of groups of finite Morley rank. In the 
present article we will only explore this approach in the opposite case, under the hypothesis of generic multiple transitivity, where our soft bounds are very loose, and one should expect very good bounds by more concrete methods.

Definition 2.5. Let $(G, \Omega)$ be a permutation group of finite Morley rank with $\Omega$ of Morley degree 1. Let $o_{k}$ denote the generic rank of an orbit for the group $G_{\alpha}{ }^{\circ}$ where $\alpha$ is an independent $k$-tuple of generic elements of $\Omega$. That is, the following set should be generic in $\Omega$ :

$$
\left\{\omega \in \Omega: \operatorname{rk}\left(\omega^{G_{\alpha}{ }^{\circ}}\right)=o_{k}\right\}
$$

We would get the same values without taking connected components, but in fact it is the connected components of the stabilizers which interest us.

Lemma 2.6. Let $(G, \Omega)$ be a primitive permutation group of finite Morley rank, $k \geq 1$, and suppose that $0<o_{k}<\operatorname{rk}(\Omega)$. Then $o_{k+1}<o_{k}$.

Proof. Assume the contrary, $o_{k+1}=o_{k}$. Our idea is then that the orbits of a stabilizer of a sequence of length $k+1$ of independent generic elements do not really depend on the last of the elements, or for that matter any of them, and hence there should be a corresponding $G$-invariant equivalence relation after eliminating spurious dependencies. This is reminiscent of various standard lines of argument in stability theory and may well be a special case of one of them.

We use the symbol $\approx$ to denote generic equality for definable sets: so $A \approx B$ means that $\operatorname{rk}(A \Delta B)<\operatorname{rk}(A), \operatorname{rk}(B)$, and in particular $\operatorname{rk}(A)=\operatorname{rk}(B)$. We also use the generic quantifier $\forall^{*} x$ with the meaning "for a strongly generic set of $x$ ". Let $r_{0}=\operatorname{rk}(\Omega)$. Our first claim is the following.

For all sequences $\alpha, \beta$ of either $k$ or $k+1$ independent generic elements of $\Omega$ we have

$$
\forall^{*} x \in \Omega \quad\left[G_{\alpha}^{\circ} \cdot x \approx G_{\beta}^{\circ} \cdot x\right]
$$

Consider the bipartite graph $\Gamma$ whose vertices are sequences of length $k$ or $k+1$ of independent generic elements of $\Omega$, with edges corresponding to inclusion or reverse inclusion. As this graph is connected, and the displayed condition in (1) defines an equivalence relation on arbitrary sequences $\alpha, \beta$ of elements from $\Omega$, it suffices to verify that condition (1) holds for a pair of vertices $\alpha, \beta$ in $\Gamma$ joined by an edge. But this is immediate as the orbits in question have Morley degree one and (generically) equal rank. So (1) holds.

Now we define an equivalence relation $E(x, y)$ on $\Omega$ as follows:

$$
\forall^{*} \alpha \in \Omega^{k}\left(G_{\alpha}{ }^{\circ} \cdot x=G_{\alpha}{ }^{\circ} \cdot y\right)
$$

This is a definable $G$-invariant equivalence relation on $\Omega$. An equivalent condition is the following, writing $\Omega^{(k)}$ for the set of sequences of $k$ independent generic elements of $\Omega$.

$$
\exists \alpha \in \Omega^{(k)} \quad(\alpha \downarrow x, y) \&\left(G_{\alpha}^{\circ} \cdot x=G_{\alpha}{ }^{\circ} \cdot y\right)
$$

Indeed, as the type over $x, y$ of a sequence of generic elements of $\Omega$ independent over $x, y$ is unique, if $G_{\alpha}{ }^{\circ} \cdot x=G_{\alpha}{ }^{\circ} \cdot y$ for one such sequence $\alpha$, then the same holds for all such sequences. 
We will write $E_{x}$ for the $E$-equivalence class of $x$. The next point is really part of a larger topic (finiteness of weight) but can be dealt with in an ad hoc way here. Cf. $[8, \S 5.6]$

$$
\forall^{*} x \operatorname{rk}\left(E_{x}\right) \geq o_{k}
$$

Let $\alpha^{(i)}$ be a sequence of $2 r_{0}+1$ independent generic elements of $\Omega^{(k)}$. Then for any pair $x, y \in \Omega$ there is an $i \leq 2 r_{0}+1$ such that $x y \downarrow \alpha^{(i)}$.

Take $x \in \Omega$ generic over $\alpha^{(1)} \ldots \alpha^{2 r_{0}+1}$, and let $C=\bigcap_{i \leq 2 r_{0}+1} G_{\alpha^{(i)}}{ }^{\circ} \cdot x$. Then by $(1) \operatorname{rk}(C)=o_{k}$. Choose $y \in C$ with $\operatorname{rk}(y / x) \geq o_{k}$. Choose $i$ so that $x, y \downarrow \alpha^{(i)}$. Then $E(x, y)$ holds by our second criterion. As $E_{x}$ is $x$-definable and contains a point $y$ of rank at least $o_{k}$ over $x, \operatorname{rk}\left(E_{x}\right) \geq o_{k}$. This proves (2).

$$
\forall^{*} x \operatorname{rk}\left(E_{x}\right) \leq o_{k}
$$

Take $x \in \Omega$ generic and let $C=E_{x}$. Choose $y \in C$ generic and independent from $x$ over the parameter $C$. Then $\operatorname{rk}(C)=\operatorname{rk}(y /$ " $C$ ") (there is a definable set $C$ on the left side, and a type over the "element" $C$ on the right). Take $\alpha \in \Omega^{(k)}$ with $\alpha \downarrow x, y$. Then $y \in G_{\alpha} \cdot x$.

As the element " $C$ " is definable from $x$, and $x, y \downarrow \alpha$, we have $y \downarrow{ }_{x}$, "C $" \alpha$. But $y \downarrow$ "C" $x$, so

$$
y \underset{\text { "C" }}{\downarrow} \alpha, x
$$

In other words, $\operatorname{rk}(y / \alpha x)=\operatorname{rk}(y / " C ")$. Therefore $\operatorname{rk}(C)=\operatorname{rk}(y / " C ")=\operatorname{rk}(y / \alpha x) \leq$ $\operatorname{rk}\left(G_{\alpha}{ }^{\circ} \cdot x\right)=o_{k}$.

Proof of Proposition 2.2. The claim is

$$
r \tau(r) \leq \rho(r) \leq r \tau(r)+\left(\begin{array}{l}
r \\
2
\end{array}\right)
$$

Let $t=\tau(r)$ and let $(G, \Omega)$ be primitive and generically $t$-transitive with $\Omega$ of rank $r$. Our claim is that

$$
r t \leq \operatorname{rk}(G) \leq r t+\left(\begin{array}{l}
r \\
2
\end{array}\right)
$$

The first inequality is immediate as $\Omega^{t}$ has rank $r t$.

Now by the definition of $\tau,(G, \Omega)$ is not generically $(t+1)$-transitive and therefore $o_{t}<\operatorname{rk}(G)$. Hence by repeated application of the foregoing lemma, $o_{t-1+i} \leq$ $\operatorname{rk}(G)-i$ until $o_{t-1+i}$ becomes 0 . In particular $o_{t-1+r}=0$.

Now let $\alpha$ be a sequence of independent generic elements of $\Omega$, of length $t+r-1$. Then $G_{\alpha}{ }^{\circ}$ has finite orbits, generically, hence fixes a generic set of points in $\Omega$, and by Lemma 1.6 we find $G_{\alpha}=1$, and $\operatorname{rk}\left(G_{\alpha}\right)=0$.

Now writing $G_{i}$ for the connected component of the pointwise stabilizer of $\left(\alpha_{1}, \ldots, \alpha_{i}\right)$ for $i \leq t+r-1$ (beginning with $\left.G_{0}=G^{\circ}\right)$, we find

$$
\operatorname{rk}(G)=\sum_{i<t+r-1} \operatorname{rk}\left(G_{i} / G_{i+1}\right)
$$

Furthermore $\operatorname{rk}\left(G_{i} / G_{i+1}\right)=\operatorname{rk}\left(\alpha_{i+1}^{G_{i}}\right)=o_{i}$, so

$$
\operatorname{rk}(G) \leq \sum_{i \geq 0} o_{i} \leq r t+\sum_{0 \leq i \leq r-1}(r-1-i)=r t+\left(\begin{array}{l}
r \\
2
\end{array}\right)
$$




\section{THE DEFINABLE SOCLE}

Using some very general results on the definable socle of a definably primitive permutation group [14], we will eventually reduce the proof of Theorem 1 to the following Theorem 2. The material in [14] is really the beginning of the subject, and if we have addressed other more specialized matters first, it was only to get them out of the way. We will not give a full account of [14] here, but certainly everything there needs to be taken into account and it would also be desirable to continue that analysis further, if possible, at some more substantial level of generality than we envision here. But the MPOSA tool in its present form is more than adequate for applications of the sort we consider here, and here we focus more on the other problems which arise in such cases.

Theorem 2. There is a function $\sigma$ which bounds the rank of a simple group of finite Morley rank in terms of the rank of any set on which it acts definably and faithfully.

Because of the simplicity of $G$, it will suffice to prove Theorem 2 in the special case when the group in question is transitive; moreover, in view of Corollary 1.19 we can assume that the action is definably primitive.

Again, this reduces via Proposition 2.2 to the following special case.

Proposition 3.1. There is a function $\tau^{\prime}$ which bounds the degree of generic multiple transitivity of a simple definably primitive permutation group of finite Morley rank in terms of the rank of the set on which it acts.

Notice again that the words "definably simple" in the formulation of Proposition 3.1 can be omitted in view of Corollary 1.19 .

We will review the necessary information from [14] and then make the reduction of Theorem 1 to Theorem 2 (and hence to Proposition 3.1).

3.1. The main case division. We now exploit the structure of the socle in a definably primitive permutation group of finite Morley rank. A good deal is known about the possibilities here, and for full details we refer to [14]. Here we emphasize those points that give immediate information relevant to bounds on rank, using comparatively soft methods, though certainly not as soft as those of the previous section. This involves the definable socle, which is the subgroup generated by its minimal definable normal subgroups. In any group $G$ of finite Morley rank, it is easy to see that the definable socle is itself definable, and is a finite direct product of finitely many simple groups and an abelian group. If there is no abelian normal subgroup then the socle contains its own centralizer. In particular, if $G$ is infinite, then the definable socle is infinite, and is a direct product of a connected normal subgroup of $G$ (the connected socle) with a finite normal subgroup of $G$, the latter commuting with $G^{\circ}$.

In definably primitive groups the situation is considerably tighter. To begin with we have the following, which is largely the preamble to Theorem 1.1 of [14].

Theorem 3 ([14, 1.1]). Let $(G, \Omega)$ be a definably primitive permutation group of finite Morley rank with $\Omega$ infinite. Let $B$ be the definable socle of $G$. Then one of the following occurs. 
(1) Affine type: $B$ is abelian, is the unique minimal normal definable subgroup of $G$, and acts regularly on $\Omega$. $G$ splits as $B \rtimes G_{\alpha}$ for any $\alpha \in \Omega$ and the action of $G$ on $\Omega$ is equivalent to the action of $G$ on $B$ with $B$ acting by translation and $G_{\alpha}$ by conjugation. Furthermore, $B$ is either torsion free divisible, or an elementary abelian p-group, and there is no nontrivial $G_{\alpha}$-invariant definable subgroup in $B$.

(2) $B$ is a finite direct product $T_{1} \times \cdots \times T_{k}$ of isomorphic connected simple groups.

What is missing in the above summary is the further subdivision of the case 2 into three further subcases, each obtained from wreath products of a corresponding basic type. These types may be referred to suggestively as regular simple type, nonregular almost simple type, and simple diagonal action, and are listed as types 2,3 , and 4(a) in [14]; here type 1 is the affine type, and type 4(b) consists of wreath products of types 2,3 , or 4 (a) as well as certain intermediate permutation groups lying between the specified socle and the full wreath product. The further results (1.2-1.4) of [14] then cast additional light on the individual types.

We will in fact need a little more from [14] but we will first see how far Theorem 3 takes us.

We make a few comments on the proof of the statement as we have given it. Note first that by definable primitivity, any nontrivial definable normal subgroup acts transitively on $\Omega$. It follows easily that if $G$ has an abelian normal subgroup then any minimal abelian normal subgroup $A$ acts regularly and the group splits as described in case (1); furthermore the definable socle centralizes $A$ and hence also acts regularly, forcing the socle to reduce to $A$. So one may suppose that all minimal normal subgroups are nonabelian. Furthermore, on general principles each nonabelian minimal definable normal subgroup of $G$ has the structure indicated in (2), so we need only consider the case in which there is more than one minimal definable normal subgroup of $G$, and they are all nonabelian. In particular, as they commute and act transitively, it follows that each acts regularly. Then for any two minimal normal subgroups $H_{1}, H_{2}$ of $G$ it is easy to see that the point stabilizer $\left(H_{1} H_{2}\right)_{\alpha}$ is the graph of an isomorphism of $H_{1}$ with $H_{2}$ (and this is the starting point for a more careful analysis of $B_{\alpha}$ ).

3.2. The torsion-free divisible case. We give a first indication that this case division is helpful for our present concerns.

Lemma 3.2. Let $(G, \Omega)$ be a definably primitive permutation group whose socle $A$ is torsion free divisible, and let $r=\operatorname{rk}(\Omega)$. Then

$$
\operatorname{rk}(G) \leq r^{2}+r
$$

Proof. This is very much like saying $\operatorname{dim}(\operatorname{AGL}(V)) \leq \operatorname{dim}(V)^{2}+\operatorname{dim}(V)$ and really has the same proof.

By Theorem 3 we can identify $\Omega$ with $A$ and $G$ with $A \rtimes G_{0}$ (note that $G_{0}$ is actually the point stabilizer of 0 under this identification).

Choose a sequence $\left(a_{1}, a_{2}, \ldots\right)$ as long as possible with $a_{i+1} \notin d\left(a_{1}, \ldots, a_{i}\right)$ (in particular $\left.a_{1} \neq 0\right)$. Let $A_{i}=d\left(a_{1}, \ldots, a_{i-1}\right)$. As the $A_{i}$ are also torsion free and divisible, $\operatorname{rk}\left(A_{i}\right) \geq i$ and therefore the sequence $\left(a_{1}, \ldots\right)$ is finite of length $n \leq r$. 
Now as $A_{n}=A$, the point stabilizer $G_{a_{1}, \ldots, a_{n}}$ is trivial. Hence writing $G_{i}$ for the point stabilizer $G_{a_{1}, \ldots, a_{i}}$ we have

$$
\operatorname{rk}\left(G_{0}\right)=\sum_{1}^{n} \operatorname{rk}\left(G_{i} / G_{i-1}\right) \leq n r \leq r^{2}
$$

and our claim follows.

More to the point is the question as to why this doesn't work in other cases. In the case of elementary abelian socles what is missing is a linear structure to bound the "dimension" (in the sense implicit above) in terms of the rank. In the case of simple socles this argument actually works quite well, as we will see next, but only bounds the rank of $G$ in terms of the rank of the socle, which must still be related to the rank of $\Omega$, since the socle usually does not act regularly.

3.3. Simple socles. Let us extract what we can from the last argument for the case of simple socles, and more generally for nonabelian socles.

Lemma 3.3. Let $S$ be an $\aleph_{0}$-saturated simple group of finite Morley rank and rank $n$. Then there are elements $a_{0}, \ldots, a_{n}$ which definably generate $S$ in the sense that

$$
S=d\left(a_{0}, \ldots, a_{n}\right)
$$

Notice that we need the saturation hypothesis even in the algebraic case: in positive characteristic there are many locally finite simple algebraic groups.

Proof. We claim that we can choose a sequence $\left(a_{0}, a_{1}, \ldots\right)$ so that setting $S_{i}=$ $d\left(a_{0}, a_{1}, \ldots, a_{i}\right)$ we have $\operatorname{rk}\left(S_{i}\right) \geq i$ until $S_{i}=S$. This will certainly prove the lemma, padding the sequence if necessary.

We begin with the case $i=1$, which requires us to choose the two elements $a_{0}, a_{1}$. If $G$ does not have bounded exponent then by the saturation hypothesis we can choose $a_{0}=a_{1}$ to be an element of infinite order. If $G$ does have bounded exponent than for the purposes of this step we may work in a minimal connected simple definable section $\bar{S}$ and lift back to $S$ afterward.

Now as $\bar{S}$ is minimal simple and of bounded exponent it follows easily that its Borel subgroups are nilpotent, and thus $\bar{S}$ is a so-called "bad group". For these there is some useful structural information and in particular any nontrivial finite subgroup lies in a unique Borel subgroup [7]. So if we take $a_{0}, a_{1}$ to be nontrivial elements in two distinct Borel subgroups then $d\left(a_{0}, a_{1}\right)$ is infinite, as required.

With the base of the induction out of the way, we continue as follows. We suppose that $S_{i}$ has rank at least $i$, with $i \geq 1$, and we wish to choose $a_{i+1}=a$ in $S$ so that

$$
\operatorname{rk}\left(d\left(S_{i}, a\right)\right)>\operatorname{rk}\left(S_{i}\right)
$$

If we cannot do this, then we find

$$
d\left(S_{i}, a\right)^{\circ}=S_{i}^{\circ}
$$

for all $a \in S$, and hence $S_{i}{ }^{\circ} \triangleleft S$, forcing $S_{i}{ }^{\circ}=S$, and we are already done.

And now as before one has the following conclusion.

Lemma 3.4. Let $G$ be a group of finite Morley rank and suppose that $G$ has a simple definable socle $S$ of rank $n$. Then $\operatorname{rk}(G) \leq n^{2}+n$. 
Proof. We can assume without loss of generality that $G$ and $S$ are $\aleph_{0}$-saturated. Consider the action of $G$ on $S$ by conjugation, which is faithful. Let the sequence $\left(a_{0}, a_{1}, \ldots, a_{n}\right)$ be chosen in accordance with the previous lemma. Then the point stabilizer of the sequence fixes $S$ pointwise and is therefore trivial, so we may repeat the computation of Lemma 3.2.

We have not yet considered the case of a general nonabelian socle, and we will return to this below.

3.4. Elementary abelian socles. Now we take up the case of an elementary abelian socle. For the present subsection, let us fix the notation as follows.

Notation 3.5. $(H, A)$ is a pair consisting of an infinite group $H$ acting definably, definably irreducibly, and faithfully on an elementary abelian p-group $A$, and the pair has finite Morley rank.

We now apply the version of Zilber's field theorem given in [7, Theorem 9.5].

Theorem 4. Let $V \rtimes G$ be a connected group of finite Morley rank with $V, G$ definable, $V$ abelian and $G$-minimal, and $C_{G}(V)=1$. Suppose that $G$ has an infinite definable abelian normal subgroup $K$. Then $C_{V}(G)=1, K$ is central in $G$, and there is an interpretable algebraically closed field over which $V$ becomes a finite dimensional vector space, $K$ becomes a group of scalars generating the field, and $G$ acts linearly.

Applying this in the present instance we get the following.

Lemma 3.6. Suppose that $H^{\circ}$ has a nontrivial definable connected abelian normal subgroup and let $K$ be a minimal such subgroup. Then there is a definable $H^{\circ}$ invariant subgroup $A_{0}$ of $A$, and a finite subgroup $K_{0}$ of $K$, such that $K / K_{0}$ acts freely on $A_{0}$ and $A$ is a direct sum of $H$-conjugates of $A_{0}$. Furthermore $K$ is central in $H^{\circ}$.

Proof. Let $A_{0} \leq A$ be a minimal definable connected $H^{\circ}$-invariant subgroup. Then $A$ is the sum of the conjugates of $A_{0}$ under $H$. In particular at least one of the conjugates of $A_{0}$ is not centralized by $K$, and we may take $A_{0}$ to have this property. Then $K_{0}=C_{K}\left(A_{0}\right)$ is finite by the minimality of $K$.

Let $\bar{H}^{\circ}=H^{\circ} / C_{H^{\circ}}\left(A_{0}\right)$. Apply the previous theorem to $A_{0} \rtimes H^{\circ} / C_{H^{\circ}}\left(A_{0}\right)$, with the subgroup $\bar{K} \cong K / C_{K}\left(A_{0}\right)$. Then $\left[K, H^{\circ}\right] \leq K_{0}$ and by connectedness $K$ is central in $H^{\circ}$.

Furthermore there is a vector space structure on $A_{0}$ with respect to which $\bar{K}$ acts by scalars and $H^{\circ} / C_{H^{\circ}}\left(A_{0}\right)$ acts linearly.

This setup produces the natural bound on the rank of $H$ when $H^{\circ}$ has a nontrivial connected abelian normal socle.

Lemma 3.7. Suppose $H^{\circ}$ has a nontrivial definable connected abelian normal subgroup. Then $\operatorname{rk}\left(H^{\circ}\right) \leq \operatorname{rk}(A)^{2}$.

Proof. Let $K$ be a minimal definable connected abelian normal subgroup of $H^{\circ}$ and let $A_{0}$ be a minimal definable connected $H^{\circ}$-invariant subgroup of $A$.

Let $r=\operatorname{rk}(A), r_{0}=\operatorname{rk}\left(A_{0}\right)$, and let $A$ be the sum of $k H$-conjugates of $A_{0}$ and no fewer. By the minimality of $A_{0}, A$ modulo a finite subgroup is a direct sum of $k$ subgroups of rank $r_{0}$ and thus $r=k r_{0}$. 
The group $\bar{H}^{\circ}=H^{\circ} / C_{H^{\circ}}\left(A_{0}\right)$ acts as a linear group on $A_{0}$. If $A_{0}$ has dimension $d$ then $\operatorname{rk}\left(\bar{H}^{\circ}\right) \leq d^{2}\left(r_{0} / d\right)=r_{0} d$. Now $H^{\circ}$ embeds into the direct product of $k$ groups definably isomorphic to $\bar{H}^{\circ}$ and hence $\operatorname{rk}(H)=\operatorname{rk}\left(H^{\circ}\right) \leq k r_{0} d=r d \leq$ $r^{2}$.

We still have to deal with the very reasonable possibility that the definable socle of $H^{\circ}$ is semisimple. In this case what is needed, initially, is a bound on the number of simple factors. For this we need the following preparation, which says, roughly speaking, that tori tend to act generically freely, thereby bounding their ranks. Here the groups playing the role of tori are the definable hulls of divisible abelian torsion subgroups, taken modulo any definable torsion free subgroups.

Lemma 3.8. Let $(G, \Omega)$ be a transitive permutation group of finite Morley rank, $T$ a definable divisible abelian subgroup of $G$, and $O(T)$ the largest definable torsion free subgroup of $T$. Then $\operatorname{rk}(T / O(T)) \leq \operatorname{rk}(\Omega)$.

Proof. Let $T_{0}$ be the maximal torsion subgroup of $T$. Note that $T_{0}$ is a countable subgroup of $T$. Therefore in a saturated model we may take a point $\alpha \in \Omega$ generic over $T_{0}$. Suppose that a torsion element $t \in T_{0}$ fixes $\alpha$. Then $t$ fixes a generic subset of $\Omega$ pointwise. Since $\Omega$ has Morley degree 1 , by Lemma 1.6 , we find $t=1$. In other words, the point stabilizer $T_{\alpha}$ is torsion free and thus contained in $O(T)$. Hence $\operatorname{rk}(T / O(T)) \leq \operatorname{rk}\left(T / T_{\alpha}\right) \leq \operatorname{rk}(\Omega)$.

For the next step, we need a result of Wagner on fields of finite Morley rank in positive characteristic.

Theorem 5 ([21], cf. [2]). Let $F$ be a field of finite Morley rank of even type and positive characteristic. Then every definable subgroup of $F^{\times}$is the definable hull of its torsion subgroup.

Accordingly we make the following definition.

Definition 3.9. A divisible abelian group of finite Morley rank is called a good torus if every definable subgroup is the definable hull of its torsion subgroup.

The preceding theorem has the following consequence. Recall that a unipotent subgroup in a group of finite Morley rank is a solvable definable connected subgroup of bounded exponent. Such groups are in fact nilpotent. There are also "characteristic 0" versions of unipotence but we do not use them here; our unipotence is $\pi$-unipotence for some finite set of primes $\pi$.

Lemma 3.10. Let $H$ be a connected solvable group of finite Morley rank acting faithfully on a unipotent group $V$ of bounded exponent. Let $U(H)$ be the maximal unipotent subgroup of $H$. Then $H / U(H)$ is a good torus.

Proof. Let $1=V_{0}<V_{1}<\cdots<V_{n}=V$ be a chain of definable $H$-invariant subgroups of $V$ such that successive quotients $A_{i}=V_{i} / V_{i-1}$ are $H$-minimal, that is infinite and without proper definable $H$-invariant subgroups. It is easy to see that the joint kernel of all the actions of $H$ on the $A_{i}$ is $U(H)$. Thus we reduce easily to the case in which $V$ is $H$-minimal and $U(H)=1$, replacing $V$ by the $A_{i}$ and $H$ by its various quotients $H / C_{H}\left(A_{i}\right)$.

Now $V$ is contained in the Fitting subgroup of $V H$ and is self-centralizing in $V H$, so the connected component $F^{\circ}(V H)$ of the Fitting subgroup of $V H$ is unipotent, and hence $F^{\circ}(V H)=V$ since $U(H)=1$. But by the structure theory for 
connected solvable groups, $V H / F^{\circ}(V H)$ is divisible abelian and thus $H$ is divisible abelian. Now Zilber's Field Theorem applies and $H$ acts on $V$ like a subgroup of the multiplicative group of a field. By Wagner's theorem, it is a good torus.

Lemma 3.11. Let $K=L_{1} \times \cdots \times L_{n}$ be a product of infinite simple groups acting faithfully on an elementary abelian p-group $V$, with the pair $(V, K)$ having finite Morley rank. Then $n \leq \operatorname{rk}(V)$.

Proof. We argue by induction on the rank of $V$. If $\operatorname{rk}(V)=0$, then $V$ is finite and an infinite simple group $K$ cannot act on $V$ faithfully. Therefore we can assume that $\operatorname{rk}(V)>0$ and $n>1$. Easily $K$ is faithful on $V^{\circ}$ so we may suppose $V$ is connected. We may also suppose that $C_{V}(K)=1$. If $V$ is not definably $K$-irreducible and $V_{0}$ is a minimal nontrivial $K$-invariant subgroup, then $V_{0}$ is infinite and connected and induction easily yields our claim. So we suppose $V$ is definably $K$-irreducible. Then $(V \rtimes K, V)$ is a primitive permutation group.

Suppose first that

$$
L=L_{n} \text { contains a nontrivial unipotent subgroup } U \text {. }
$$

Then $W=C_{V}{ }^{\circ}(U)<V$ is nontrivial.

Let $K_{1}=L_{1} \times \cdots \times L_{n-1}$. Then $K_{1}$ acts on $W$ and if the action is faithful we find $n-1 \leq \operatorname{rk}(W)<\operatorname{rk}(V)$ and our claim follows.

So we may suppose that $L_{1}$ acts trivially on $W$. Let $V_{1}=C_{V}\left(L_{1}\right)<V$. This contradicts the definable $K$-irreducibility of $V$.

So now we consider the alternative, which we may take to be the following.

$$
\text { No factor } L_{i} \text { contains a nontrivial unipotent subgroup }
$$

Now if $B$ is a Borel subgroup of any factor $L_{i}$, then $U(B)=1$ and by the preceding lemma $B$ is a good torus. Since a product of good tori is a good torus [1, Lemma 4.21] we can find a good torus $T$ in $K$ of rank at least $n$. Then $O(T)=1$ and thus by Lemma $3.8 \operatorname{rk}(T) \leq \operatorname{rk}(V)$. So $n \leq \operatorname{rk}(V)$ as claimed.

Now we use this information to give a bound on the rank of $H$ in terms of the ranks of $A$ and the ranks of the definable simple nonabelian subgroups of $H^{\circ}$.

Lemma 3.12. Suppose that every definable simple nonabelian subgroup of $H^{\circ}$ has rank at most $s$, and $r=\operatorname{rk}(A)$. Then the rank of $H$ is at most

$$
\max \left(r^{2}, r\left(s^{2}+s\right)\right)
$$

Proof. In view of Lemma 3.7 we will suppose that all minimal normal definable subgroups of $H^{\circ}$ are simple. Let there be $n$ such. For $L$ a definable normal simple subgroup of $H^{\circ}$, let $H_{L}=H^{\circ} / C_{H^{\circ}}(L)$. Then there is an embedding $H^{\circ} \rightarrow \prod_{L} H_{L}$ and thus $\operatorname{rk}\left(H^{\circ}\right) \leq n \max _{L}\left(\operatorname{rk}\left(H_{L}\right)\right)$

Now identify $\bar{L}$ with its image in $H_{L}$. Then $L$ is a definable simple normal subgroup of $H_{L}$, and we claim that $L$ is the definable socle of $H_{L}$. Let $K$ be the preimage in $H^{\circ}$ of $C_{H_{L}}(L)$. Then $[K, L] \leq C_{L}(L)=1$, so $K=C_{H^{\circ}}(L)$. Thus $L$ is self-centralizing in $H_{L}$ and is the definable socle.

So by Lemma 3.4 we have $\operatorname{rk}\left(H_{L}\right) \leq s^{2}+s$ and thus $\operatorname{rk}(H) \leq n\left(s^{2}+s\right) \leq r\left(s^{2}+s\right)$, taking into account Lemma 3.11.

Now we go back to the case of nonabelian socles and make a similar reduction. 
3.5. Nonabelian socles. Throughout the present subsection $(G, \Omega)$ denotes a permutation group of finite Morley rank with nonabelian definable socle. At this point one can usefully examine the subdivision into cases afforded by the full statement of MPOSA, but we continue on with less precise methods. Set $r=\operatorname{rk}(\Omega)$.

Lemma 3.13. If $G$ has more than one minimal definable normal subgroup, then

$$
\operatorname{rk}(G) \leq r^{2}+2 r
$$

Proof. Let $L$ be a minimal normal subgroup of $G$. By our hypothesis $C_{G}(L)$ is nontrivial and hence by definable primitivity $L$ and $C_{G}(L)$ both act transitively on $\Omega$. It follows that each acts regularly and in particular $\operatorname{rk}\left(C_{G}(L)\right)=r$, so $\operatorname{rk}(G) \leq r+\operatorname{rk}\left(G / C_{G}(L)\right)$.

Now write $L=L_{1} \times \cdots \times L_{n}$ with the $L_{i}$ isomorphic definable simple normal subgroups of $G^{\circ}$. As $L$ acts regularly on $\Omega$ we have $\operatorname{rk}\left(L_{i}\right)=r / n$. Then Lemma 3.4 yields

$$
\operatorname{rk}\left(G / C_{G}(L)\right) \leq n\left((r / n)^{2}+(r / n)\right)=r^{2} / n+r
$$

and our claim follows.

Now suppose that $G$ contains a unique minimal definable normal subgroup $L$, which is nonabelian. We should note that if $G$ were connected then that subgroup would in fact be simple, as $G$ acts transitively on the simple factors of a nonabelian minimal normal subgroup, and then the situation would be quite transparent. But what this suggests, correctly, is that primitive groups will have a strong tendency to be disconnected, in general.

In this case we finally need to use the more precise information from [14], describing the point stabilizer $L_{\alpha}$, which is a characteristic feature of the MPOSA point of view. There are the following two possibilities:

(a) $L$ is a product of simple factors $L_{i}$ and $L_{\alpha}=\prod_{i}\left(L_{i}\right)_{\alpha}$ (possibly $L_{\alpha}=1$ here);

(b) $L$ is a product of $\ell$ groups $L_{i}$, each of which is a product of $k$ simple factors $L_{i j}$, with $k \geq 2$, and $\left(L_{i}\right)_{\alpha}$ is a diagonal subgroup of $L_{i}$, and in particular $\operatorname{rk}\left(\left(L_{i}\right)_{\alpha}\right)$ is definably isomorphic to $L_{i j}$ for each $j$.

Lemma 3.14. If $G$ has a unique minimal normal subgroup $L$, and its simple factors have rank $s$, then corresponding to cases $(a, b)$ above we have the following estimates.

(a) $\operatorname{rk}(G) \leq r\left(s+s^{2}\right)$;

(b) $\operatorname{rk}(G) \leq 2\left(r^{2}+r\right)$

Proof. Let $L$ be the product of $n$ simple factors, each of rank $s$. Then our estimate for $\operatorname{rk}(G)$ is

$$
n\left(s^{2}+s\right)
$$

In case $(a)$ we have $n \leq r$. So we consider case $(b)$.

If $L$ acts regularly on $\Omega$ then $s=r / n \leq r$.

If the point stabilizer $L_{\alpha}$ is a product of $\ell$ diagonal subgroups then $n=k \ell$ for some $k$ and $r=\operatorname{rk}(L)-\operatorname{rk}\left(L_{\alpha}\right)=n s-\ell s=(k-1) \ell s, s=r /(k-1) \ell=r /(n-\ell) \leq$ $(2 / n) r$. So $n\left(s^{2}+s\right) \leq(4 / n) r^{2}+2 r \leq 2\left(r^{2}+r\right)$ since $n \geq 2$.

The upshot of all of this is that we need an estimate for the rank of a simple group acting on $\Omega$ in terms of the rank of $\Omega$, or, in view of Proposition 2.2, a bound on the degree of generic multiple transitivity for such an action. 
Of course, this reduction of a general problem on primitive permutation groups to the simple case is a typical application of the MPOSA point of view.

\section{Actions of Finite groups on CONNECTED SOlvable Groups}

The present section has a preparatory character. In the next section we use that fact that generically $n$-transitive groups have the symmetric group $\operatorname{Sym}(n)$ as a section. Here we examine definable actions of $\operatorname{Sym}(n)$, and related groups, on connected solvable groups of finite Morley rank, looking for lower bounds on the rank. This is an issue which has not arisen in the past and has some affinities with linear representation theory, though an action on a connected abelian group is not necessarily very closely connected with a linear representation, as far as we know. Getting sharp bounds for this sort of problem seems challenging.

There are three natural variations, all of which come into play.

Problem 8. Let $\Sigma$ be a finite group. Find lower bounds for each of the following.

(1) The minimal rank of a connected solvable group of finite Morley rank which affords a faithful representation of $\Sigma$.

(2) The minimal rank of a connected solvable group of finite Morley rank which affords a faithful representation of a central extension of $\Sigma$.

(3) The minimal rank of a connected solvable group of finite Morley rank which affords a faithful representation of a group $\hat{\Sigma}$ which covers $\Sigma$, i.e. maps homomorphically onto $\Sigma$.

We will work with abelian groups rather than solvable groups, but in most cases we can reduce to $\Sigma$-minimal groups, in which case there is no difference. But the question makes sense also more generally, without even the hypothesis of solvability.

Actually, as we will see we are interested particularly in actions of finite groups on divisible abelian groups. This brings us a little closer to the characteristic zero linear theory.

The estimate we will need is the following. This is off the main line of our discussion, and any estimate of the kind will suffice for our subsequent purposes.

Proposition 4.1. Let $A$ be a connected abelian group of finite Morley rank, and $\Sigma$ a finite group acting definably and faithfully on $A$. Suppose that $\Sigma$ maps surjectively onto $\operatorname{Sym}(n)$. Then $\operatorname{rk}(A) \geq\lfloor n / 4\rfloor$

We return to the main line in the next section.

4.1. Generalities. We dispose of some formal points before taking up anything concrete. Recall that the Frattini subgroup $\Phi(\Sigma)$ of a finite group $\Sigma$ is the intersection of its maximal subgroups, and is nilpotent.

Definition 4.2. Let $\Sigma \rightarrow \bar{\Sigma}$ be a surjection between finite groups. The map, or by abuse $\Sigma$ itself, is called a Frattini cover of $\bar{\Sigma}$ if the kernel of the map is contained in the Frattini subgroup of $\Sigma$.

An equivalent condition is this: no proper subgroup of $\Sigma$ covers $\bar{\Sigma}$. So we have a trivial but useful starting point.

Lemma 4.3. Let $\Sigma_{0}$ be a finite group, $\Sigma$ a finite group mapping homomorphically onto $\Sigma_{0}$. Then $\Sigma$ contains a subgroup $\Sigma_{1}$ which is a Frattini cover of $\Sigma_{0}$. 
For the proof just take $\Sigma_{1}$ minimal. Notice that if we also have a faithful representation of $\Sigma$ on a connected abelian group of finite Morley rank, then we have in particular a faithful representation of a Frattini cover. This has the advantage that the kernel of a Frattini cover is nilpotent, so we are getting much more control over the group. What we would prefer is to reduce even further, to central extensions.

Now we take a preliminary look at the minimal modules for our finite groups.

Lemma 4.4. Let $\Sigma$ be an almost simple group (i.e., the socle is simple). Let $A$ be a connected abelian group of minimal Morley rank such that $\Sigma$ acts faithfully and definably on $A$. Then $\Sigma$ acts faithfully on some $\Sigma$-minimal definable section of $A$.

Proof. We argue by induction on $\operatorname{rk}(A)$. Let $A_{0} \leq A$ be $\Sigma$-minimal. If $\Sigma$ acts faithfully on $A_{0}$ or $A / A_{0}$ we conclude directly or by induction, respectively. In the alternative case the socle $\Sigma_{0}$ of $\Sigma$ acts trivially on both factors and therefore each element $a \in A$ gives rise via commutation to a homomorphism $\alpha: \Sigma_{0} \rightarrow A_{0}$, from a simple group to an abelian group. So in this case $\Sigma_{0}$ acts trivially on $A$, a contradiction.

Next we prepare some general estimates for actions of elementary abelian groups.

Lemma 4.5. Let $E$ be an elementary abelian $p$-group and $m=m_{p}(A)$. If $E$ acts faithfully on the connected p-divisible abelian group $A$ of finite Morley rank, then $\operatorname{rk}(A) \geq m$.

Proof. Let $V \leq A$ be $E$-minimal. Let $E_{0}$ be the kernel of the action of $E$ on $V$, a subspace of $E$ of codimension 1. If $E_{0}$ acts faithfully on $A / V$ then we conclude by induction.

So let $E_{1}$ be the kernel of the action of $E_{0}$ on $A / V$. Then $E_{1}$ acts trivially on the factors of the chain $1<V<A$. So for $e \in E_{1}^{\#}$ commutation with $e$ gives a homomorphism $\epsilon: A / V \rightarrow A$ with image a connected elementary abelian $p$-group, contradicting our hypothesis.

4.2. Actions of symmetric groups. We aim here at lower bounds for the rank of a connected abelian group on which $\operatorname{Sym}(n)$ acts faithfully, particularly when the abelian group in question is the definable closure of a $p$-torus for some $p$. Of course, we will really need to deal with groups covering $\operatorname{Sym}(n)$ and these do not necessarily behave in the same way, so the reader may prefer to pass on to the next subsection which returns to the more general problem, with worse estimates.

Here it would be very useful to have a result of the following kind: if $T$ is the definable closure of a $p$-torus and is $F$-minimal under the action of a finite group $F$, then the corresponding Tate module is $F$-irreducible. Unfortunately this is nonsensical, as one sees already by taking $F=1$, but perhaps something can be rescued in this direction.

Before stating our next lemma we note that the symmetric group $\operatorname{Sym}(6)$ is isomorphic to the symplectic group $\operatorname{Sp}(4,2)$ and therefore has a faithful representation on a connected abelian group of Morley rank 4, namely the corresponding vector space over the algebraic closure of $\mathbb{F}_{2}$.

Lemma 4.6. Let $A$ be an abelian group on which $\operatorname{Sym}(n)$ acts definably and faithfully, with $A$ either a finite elementary abelian p-group or a connected abelian group. Let $\delta(A)$ be $m_{p}(A)$ in the first case and $\operatorname{rk}(A)$ in the second case. Then $\delta(A) \geq n-1$ unless $n=6, d=4$, and $A$ is an elementary abelian 2-group (finite or connected). 
Proof. We proceed by induction on $n$ and then by induction on $\delta(A)$. If $n=2$ our claim is vacuous. If $n \geq 3$ and $d=1$ then the transpositions must act by inversion on $G$, and the action cannot be faithful. So we may suppose throughout that $d \geq 2$ and $n \geq 4$. Write $\Sigma$ for $\operatorname{Sym}(n)$.

We treat separately the case $n=4$. Let $V$ be the Klein 4 -group in $\Sigma$. Let $A_{0}<A$ be $V$-minimal. Then $V$ has a kernel on $A_{0}$ and this kernel has three conjugates in $\Sigma$. It follows easily that $d \geq 3$. So from now on we suppose

$$
n \geq 5
$$

In particular $\Sigma$ is almost simple and $A$ may be taken to be $\Sigma$-minimal in the connected case, irreducible in the finite case. In particular $A$ is either an elementary abelian 2-group, or is 2-divisible.

We deal first with the case of an elementary abelian 2-group. We will use the connected component notation below with an eye on the case in which $A$ is connected. This operator should be interpreted as vacuous $\left(X^{\circ}=X\right.$, not $\left.X^{\circ}=1\right)$ when $A$ itself is finite.

Fix a transposition $\tau \in \Sigma$. Then $1 \leq[\tau, A] \leq C_{A}{ }^{\circ}(\tau) \leq A$. If the three sections $A_{1}=[\tau, A], A_{2}=C_{A}{ }^{\circ}(\tau) /[\tau, A]$, and $A_{3}=A / C_{A}{ }^{\circ}(\tau)$ are all nontrivial, consider the action of the group $\Sigma_{0}=C_{\Sigma}(\tau) \cong \operatorname{Sym}(n-2)$. If $n \geq 7$ so that $n-2 \geq 5$, then $\Sigma_{0}$ acts faithfully on at least one of the sections $A_{i}$ and thus we find $\delta\left(A_{i}\right) \geq n-3$, $\delta(A) \geq(n-3)+2=n-1$ as required. If $n=6$ then the socle of $\operatorname{Sym}(4)$ acts faithfully on at least one of these sections, which therefore has rank at least 2 . So we get our lower bound $\delta(A) \geq 4$ in this case. If $n=5$ we have only to deal with the case in which $\delta\left(A_{i}\right)=1$ for $i=1,2,3$. Then $\operatorname{Sym}(3)$ is generated by involutions and each of them acts trivially on each section, so $\operatorname{Sym}(3)$ acts trivially on each section and therefore an element of order 3 acts trivially on $A$, a contradiction.

This disposes of all cases in which the elementary abelian 2-group $A$ has the three sections $A_{1}, A_{2}, A_{3}$ all nontrivial. So at least one of these sections is trivial. But $\tau$ acts faithfully on $A$, so we find $C_{A}{ }^{\circ}(\tau)=[\tau, A]$ or in other words $[\operatorname{ker}(1-\tau)]^{\circ}=$ $\operatorname{im}(1-\tau)$. So $\delta\left(A_{1}\right)=\delta\left(A_{3}\right)$. If $n \geq 7$ then again $\Sigma_{0}$ acts faithfully on one of these sections and thus $\delta(A) \geq 2(n-3) \geq n-1$.

We now have to treat the small cases with $n=5,6$.

Suppose first $\delta\left(A_{1}\right)=\delta\left(A_{3}\right)=1$. Let $V$ be a 4 -group in $\Sigma$. Then $C_{A}{ }^{\circ}(V)$ is nontrivial and hence $C_{A}{ }^{\circ}(V)=C_{A}{ }^{\circ}(i)$ for $i \in V^{\#}$ or in other words $C_{A}{ }^{\circ}(i)=$ $C_{A}{ }^{\circ}(j)$ for commuting involutions $i, j$, and as $n \geq 5$ we conclude that $C_{A}{ }^{\circ}(i)=$ $C_{A}{ }^{\circ}(\Sigma)$ which may be supposed trivial. Thus we have a contradiction.

If $\delta\left(A_{1}\right)=\delta\left(A_{3}\right)=2$ and $\delta(A)=4$ then the only remaining case is $n=6$, which we allow.

So now we pass to the main case.

\section{$A$ is 2-divisible}

Recall that $A$ is either connected, or is a finite elementary abelian $p$-group with $p$ odd, now.

Consider an involution $\tau$ and let $\Sigma_{0}=C_{\Sigma}(\tau) \cong \operatorname{Sym}(n-2)$. We have

$$
A=A_{\tau}^{+}+A_{\tau}^{-}
$$

with $A_{\tau}^{+}, A_{\tau}^{-}$the subgroups centralized by or inverted by $\tau$ respectively. If $A$ is infinite (connected) then the intersection $A_{\tau}^{+} \cap A_{\tau}^{-}$is finite and these groups are connected. 
We have actions of $\Sigma_{0}$ on $A_{\tau}^{+}$and $A_{\tau}^{-}$, and at least one of these actions is faithful. So $\delta(A) \geq(n-3)+1=n-2$ with equality only if one of the factors has rank 1 and the other has rank $n-3$.

Suppose $\delta\left(A_{\tau}^{+}\right)=1$. The same holds for any transposition as they are conjugate. So if $\tau, \tau^{\prime}$ are transpositions whose product $\sigma$ has order 3, then $\tau$ and $\tau^{\prime}$ invert subgroups of corank 1 in $A$, and hence $\sigma$ inverts a subgroup of corank at most 2. So in this case $\delta(A)=2$ and thus by the above $n-3=1, n=4$. Then consider a pair of commuting transpositions $\tau_{1}, \tau_{2}$. We have $A_{\tau_{1}}^{ \pm}=A_{\tau_{2}}^{ \pm}$in some order and hence $\tau_{1} \tau_{2}$ acts either trivially or by inversion on $A$. Then the action of $\tau_{1} \tau_{2}$ commutes with the action of $\Sigma$ on $A$ and therefore this action is not faithful. We conclude

$$
\delta\left(A_{\tau}^{-}\right)=1, \delta\left(A_{\tau}^{+}\right)=n-3>1
$$

for any transposition $\tau \in \Sigma$. In particular $n \geq 5$.

For any pair of commuting transpositions $\tau, \tau^{\prime}$, the element $\tau^{\prime}$ centralizes $A_{\tau}^{-}$, as otherwise we would have $A_{\tau}^{-}=A_{\tau^{\prime}}^{-}$for all such commuting pairs and then $A_{\tau}^{-}$is independent of $\tau$, since $n \geq 5$.

For any transposition $\tau$, the action of $\tau$ on $A / A_{\tau}^{-}$is trivial and in particular any subgroup containing $A_{\tau}^{-}$is $\tau$-invariant.

Now we need to work out the action in more detail. Fix two noncommuting transpositions $\tau_{1}, \tau_{2}$ and let $A_{i}=A_{\tau_{i}}^{-}$for $i=1,2$. Take $a_{1} \in A_{1}$ and choose $a_{2} \in A_{2}$ so that

$$
a_{1}^{\tau_{2}}=a_{1}+a_{2}
$$

Let $\sigma=\tau_{2} \tau_{1}$. Then $a_{1}^{\sigma}=-a_{1}+a_{2}^{\tau_{1}}$ and $\sigma$ carries $A_{1}$ to $A_{2}$, so $a_{2}^{\tau_{1}} \in a_{1}+A_{2}$ and therefore $a_{2}^{\tau_{1}}=a_{1}+a_{2}$. Thus $a_{2}=a_{1}^{\sigma}$. Similarly $a_{2}^{\tau_{1}}=a_{2}^{\sigma^{-1}}+a_{2}=a_{1}+a_{2}$.

Now let $\tau_{i}$ be the elementary transposition $(i, i+1)$ and let $A_{i}=A_{\tau_{i}}^{-}$. Assuming $\delta(A)<n-1$, let $k$ be minimal so that $\delta\left(A_{1}+\cdots+A_{k}\right)<k$. Then $A_{k} \leq$ $A_{1}+\cdots+A_{k-1}$ and therefore $A_{1}+\cdots+A_{k}$ is $\Sigma$-invariant, so $k=n-1$ and $A=A_{1}+\cdots+A_{n-2}$ is an almost direct sum.

Consider the cycle $\sigma=(12 \ldots n)$. For any $i<n-1$ if $\sigma_{i}=\tau_{i+1} \tau_{i}$ then $\sigma \sigma_{i}^{-1}$ commutes with $\tau_{i}$ and thus $\sigma$ acts on $A_{i}$ as $\sigma_{i}$ does. This holds also for $A_{n-1}$ with a notational variation. So for $a \in A_{i}$ we have $a^{\tau_{i+1}}=a+a^{\sigma}$, and similarly $a^{\tau_{i-1}}=a+a^{\sigma^{-1}}$.

Now consider any relation

$$
a_{1}+\cdots+a_{n-1}=0
$$

with $a_{i} \in A_{i}$. Applying $\tau_{i}$ we get

$$
a_{i-1}^{\tau_{i}}-a_{i}+a_{i+1}^{\tau_{i}}=a_{i-1}+a_{i}+a_{i+1}
$$

or

$$
a_{i-1}^{\sigma}+a_{i+1}^{\sigma^{-1}}=2 a_{i}
$$

From this we easily derive

$$
i a_{i+1}=(i+1) a_{i}^{\sigma}
$$

for all $i<n-1$. For $i=1$ the equation $(*)$ reduces to the desired form and after that induction applies. But there is also a final equation for $i=n-1$ which reduces to

$$
0=n a_{n-1}^{\sigma}
$$


or $n a_{n-1}=0$. As $A$ is divisible it follows that there are only finitely many possible values for $a_{n-1}$, which contradicts our assumption.

4.3. Groups covering $\operatorname{Sym}(n)$. Now we look at actions on connected abelian groups of groups $\Sigma$ mapping homomorphically onto $\operatorname{Sym}(n)$.

We first mention some comparatively low dimensional representations of double covers of $\operatorname{Sym}(n)$, and the like. The $\operatorname{Schur}$ multiplier of $\operatorname{Sym}(n)$ is $\mathbb{Z} / 2 \mathbb{Z}$, but there are two nonisomorphic double covers.

(1) $2 . \operatorname{Sym}(4)=\mathrm{GL}(2,3)$ has a 2 dimensional representation.

(2) Both of the double covers of $\operatorname{Sym}(7)$ have 4-dimensional representations in characteristic 7 .

(3) $\operatorname{Alt}(8)=\mathrm{SL}(4,2)$ has 4-dimensional representations in characteristic 2.

Now we give our main estimate for groups covering $\operatorname{Sym}(n)$.

Proof of Proposition 4.1. We may suppose that $\Sigma$ is a Frattini cover of $\operatorname{Sym}(n)$.

Suppose first that $\operatorname{rk}(A)=1$. In this case any abelian subgroup of $\Sigma$ is cyclic and in particular the Sylow 2-subgroup $T$ of $\Sigma$ contains a unique involution. So this involution is in the kernel of the map $\Sigma \rightarrow \operatorname{Sym}(n)$, and $T$ itself is either cyclic or a generalized quaternion subgroup. So the image of $T$ in $\operatorname{Sym}(n)$ is abelian, contradicting the structure of $\operatorname{Sym}(n)$.

So we may suppose $\operatorname{rk}(A) \geq 2$. That being the case, we may also suppose $n \geq 8$, but as one might reasonably aim at a sharper lower bound, e.g. $(n-2) / 2$, we will only assume here that

$$
n \geq 5
$$

Let $\Gamma$ be the kernel of the given homomorphism $\Sigma \rightarrow \operatorname{Sym}(n)$. Since $\Sigma \rightarrow \operatorname{Sym}(n)$ is a Frattini cover, $\Gamma$ is nilpotent. Let $\Gamma_{2}$ be the Sylow 2-subgroup. If $\Gamma_{2}=1$ then one expects the same estimates as in the preceding subsection, but in any case $\Sigma$ contains an elementary abelian 2-subgroup $E$ with $m(E)=\lfloor n / 2\rfloor$ and this suffices for our estimate. So we suppose

$$
\Gamma_{2}>1
$$

Let $A_{0} \leq A$ be $\Sigma$-minimal. Let $\Gamma_{0}$ be the kernel of the action of $\Sigma$ on $A_{0}$. If $\Gamma_{0}$ is contained in $\Gamma$ then we can look at the action of $\Sigma / \Gamma_{0}$ on $A_{0}$ and conclude by induction unless $A=A_{0}$. On the other hand, if $\Gamma_{0}$ is not contained in $\Gamma$ then $\Gamma_{0}$ covers $\operatorname{Alt}(n)$ and hence $\Sigma$ is generated by $\Gamma_{0}$ and a 2-element, and $\Sigma^{\prime} \leq \Gamma_{0}$. If $A_{0}<A$ we can consider the action of $\Sigma$ on $A / A_{0}$ similarly and conclude by induction unless $\Sigma^{\prime}$ acts trivially on $A / A_{0}$. So finally $\Sigma^{\prime}$ acts trivially on $A_{0}$ and $A / A_{0}$ and hence the subgroup of $\Sigma^{\prime}$ generated by its $2^{\prime}$-elements acts trivially on $A$, a contradiction as this covers $\operatorname{Alt}(n)$. So we may suppose that $A$ is $\Sigma$-minimal.

Now let $\Sigma_{1}$ be any subgroup of $\Sigma$ covering $\operatorname{Alt}(n)$. We claim that $A$ is $\Sigma_{1}$ minimal. We may replace $\Sigma_{1}$ by a minimal subgroup covering $\operatorname{Alt}(n)$ and then $\Sigma_{1}=\Sigma_{1}^{\prime}$ and thus $\Sigma_{1}=\Sigma^{(\infty)}$ is normal in $\Sigma$. Now suppose $A$ is not $\Sigma_{1}$-minimal. Let $A_{0}<A$ be $\Sigma_{1}$-minimal. Then $A$ is the almost direct sum of two conjugates of $A_{0}$. Thus $\operatorname{rk}(A)=2 \operatorname{rk}\left(A_{0}\right)$. Now there is a subgroup of $\Sigma_{1}$ covering a copy of $\operatorname{Sym}(n-2)$ inside $\operatorname{Alt}(n)$, so we may suppose by induction that $\operatorname{rk}\left(A_{0}\right) \geq\lfloor(n-2) / 4\rfloor$ and thus $\operatorname{rk}(A) \geq\lfloor n / 4\rfloor$ and we conclude. So we may suppose that $A$ is $\Sigma_{1}$-minimal for all such $\Sigma_{1}$.

Now we return to the structure of the nilpotent group $\Gamma$ and its Sylow 2-subgroup $\Gamma_{2}$. Suppose that $\Gamma_{2}$ is noncyclic and take $k$ minimal so that $Z_{k}\left(\Gamma_{2}\right)$ is noncyclic. Let $C=Z_{k+1}\left(\Gamma_{2}\right)$ and let $Q$ be the preimage in $\Gamma_{2}$ of $\Omega_{1}\left(Z_{k}\left(\Gamma_{2}\right) / C\right)$. Let $Q_{0}=C_{Q}(C)$. 
Now $\Gamma_{2}$ acts trivially on $Q / C$ and thus there is an action of $\operatorname{Sym}(n)$ on $Q / C$. If this action is faithful then $m_{2}(Q / C) \geq 4$ and hence $Q_{0}>C$. In this case we can consider the action of $\operatorname{Sym}(n)$ on $Q_{0} / C$, and if this is faithful then $m_{2}\left(Q_{0} / C\right) \geq n-1$ unless $n=6$ and $m_{2}\left(Q_{0} / C\right)=4$.

Suppose $m_{2}\left(Q_{0} / C\right) \geq n-1$. If $Q_{0}$ is elementary abelian then we get a strong estimate. Otherwise, $Q_{0}^{\prime} \leq C$ is the subgroup of order 2 and the commutator map $Q_{0} / C \times Q_{0} / C \rightarrow Q_{0}^{\prime}$ gives a symplectic structure on $Q_{0} / C$, possibly degenerate. We may take a totally isotropic subspace of $Q_{0} / C$ of dimension $(n-1) / 2$, which lifts back to an abelian subgroup $V \leq Q_{0}$ for which $m_{2}(V / C)=(n-1) / 2$. Thus $V$ contains an elementary abelian subgroup of rank $(n-1) / 2$ and we get the desired estimate.

Now suppose $m_{2}\left(Q_{0} / C\right)=4$ and $n=6$, with $\operatorname{Sym}(n)$ acting faithfully and with $Q_{0}^{\prime}>1$. Let $Q_{1}$ be the preimage in $Q_{0}$ of $\Omega_{1}\left(Q_{0} / Q_{0}^{\prime}\right)$. Then $Q_{1} / Q_{0}^{\prime}$ is elementary abelian of rank 4. As $\operatorname{Sym}(n)$ respects the symplectic structure it coincides with $\operatorname{Sp}(4,2)$ and in particular acts transitively on $Q_{1} / Q_{0}^{\prime}$. Then as $Q_{1} \backslash C$ contains involutions, $Q_{1}$ has exponent two and thus is elementary abelian of rank 5 . So $\operatorname{rk}(A) \geq 5$.

So we are left with the case in which $\operatorname{Alt}(n)$ centralizes $Q / C$ and hence a subgroup of $\Sigma$ covering $\operatorname{Alt}(n)$ centralizes $Q$. If $Q$ contains a noncyclic elementary abelian subgroup $E$ then let $A_{0} \leq A$ be $E$-minimal and let $E_{0}$ be the kernel of the action of $E$ on $A_{0}$. Then $C_{A}{ }^{\circ}\left(E_{0}\right)$ is $C_{\Sigma}(Q)$-invariant and thus $C_{A}{ }^{\circ}\left(E_{0}\right)=A$, implying $E_{0}=1$ and $E$ is cyclic after all.

So $Q$ contains a unique involution and is therefore either cyclic or generalized quaternion. But by our current assumption $Q$ is not cyclic. On the other hand by construction $Q / Z(Q)$ is elementary abelian, and so $Q$ is a quaternion group of order 8 , and is centralized by a subgroup of $\Sigma$ covering $\operatorname{Alt}(n)$.

Taking a 2-element $t \in \Sigma$ which represents a transposition in $\operatorname{Sym}(n)$, we may suppose after adjustment that $t$ centralizes an element $a$ of order 4 in $Q_{0}$. Then $C(a)$ covers $\operatorname{Sym}(n)$ so by minimality $a \in Z(\Sigma)$ and in particular $a \in Z(Q)$, a contradiction.

All of this shows that $\Gamma_{2}$ is cyclic. Now one can show in the same way that $\Gamma$ is cyclic and centralized by $\operatorname{Alt}(n)$ and pursue the matter further, but we will stop here with a simple estimate. We have $\Gamma_{2}$ centralized by a subgroup of $\Sigma$ covering $\operatorname{Alt}(n)$. Taking an elementary abelian subgroup of $\operatorname{Alt}(n)$ of rank at least $2\lfloor n / 4\rfloor$, and lifting to a 2-subgroup of $\Sigma$, we get an elementary abelian subgroup of rank at least $\lfloor n / 4\rfloor$ in $\Sigma$, and conclude.

\section{Simple Permutation Groups}

In the present section we will bring to bear a good deal of recent work on simple groups of finite Morley rank to the problem of bounding their rank or degree of multiple transitivity in terms of the rank of a set acted upon faithfully. We begin by recalling some of the relevant information.

5.1. The four types. The 2-Sylow theory for groups of finite Morley rank gives a broad division of all such groups into four types, according to the structure of their 2-Sylow ${ }^{\circ}$ subgroups (maximal connected 2-subgroups). In general these have the form

$$
U * T
$$


a central product with finite intersection of a 2-unipotent group $U$ (a connected, definable 2-group of bounded exponent) and a 2-torus $T$ (a divisible abelian 2group, not definable). The group $G$ is said to be of even, odd, mixed, or degenerate type according as $S=U, S=T, U, T>1$, or $S=1$. A delicate analysis depending on a body of technology developed in the finite case, and some other ingredients, shows the following.

Theorem 6. A simple group of finite Morley rank of even type is algebraic.

For a group $G$ of finite Morley rank, let $T_{2}(G)$ be the subgroup generated by all definable hulls of 2-tori in $G$, and $U_{2}(G)$ the subgroup generated by all unipotent 2-subgroups.

Theorem 7. Let $G$ be a group of finite Morley rank. Then normal subgroups $T_{2}(G)$ and $U_{2}(G)$ have odd and even types respectively. Furthermore, $T_{2}(G)$ and $U_{2}(G)$ commute, with $G /\left[T_{2}(G) * U_{2}(G)\right]$ of degenerate type.

Theorem 7 depends on Theorem 6 . In fact what one actually shows is that Theorem 7 holds for groups whose definable simple sections of even type are all algebraic.

These theorems have the following consequence, which can however be proved much more directly [6].

Theorem 8. Let $G$ be a simple group of finite Morley rank containing no nontrivial divisible torsion subgroup. Then $G$ is of degenerate type.

Another result of a general character with a comparatively short proof is the following [5].

Theorem 9. Let $G$ be a connected group of finite Morley rank of degenerate type. Then $G$ contains no involutions.

Actually this can be extended to a similar result holding for any prime $p$. In that form it reads as follows.

Theorem 10 ([5]). Let $G$ be a connected group of finite Morley rank containing no infinite abelian p-subgroup. Then $G$ contains no elements of order $p$.

The classification of simple groups of even type has various structural consequences which are probably not accessible without that classification. For example we have the following [1].

Proposition 5.1. Let $G$ be a connected group of finite Morley rank of even type and suppose that $G$ contains no nontrivial normal 2-unipotent subgroup. Then $G$ is a central product of definable subgroups

$$
E(G) * \hat{O}(G)
$$

where $E(G)$ is a product of quasisimple algebraic groups in characteristic two, and $\hat{O}(G)$ has degenerate type.

These notions apply in our context as follows.

Lemma 5.2. Let $G$ be a definably primitive permutation group of finite Morley rank. Then $G^{\circ}$ is not of mixed type. 
Proof. Supposing the contrary, $U_{2}(G)$ is nontrivial by Theorem 7 . If $G$ contains a nontrivial normal 2-unipotent subgroup then, in view of definable primitivity of $G$, the definable socle of $G$ is an elementary abelian 2-group. But then the definable socle of $G$ is disjoint from $T_{2}(G)$ and thus $T_{2}(G)$ is trivial.

Similarly, if $G$ contains no nontrivial normal 2-unipotent subgroup, then the definable socle of $U_{2}(G)$ is a product of simple groups of even type and thus the definable socle of $G$ is also a product of simple groups of even type, and in fact all of the factors are isomorphic. Again, this forces the socle to miss $T_{2}(G)$ and thus $T_{2}(G)=1$.

We can refine this a little when we have generic 2-transitivity as well.

Lemma 5.3. Let $(G, \Omega)$ be a definably primitive and generically 2-transitive permutation group of finite Morley rank. Then $G^{\circ}$ is either of odd or of even type.

Proof. We have already eliminated mixed type. On the other hand, as $G$ is generically 2-transitive, also $G^{\circ}$ is generically 2 -transitive, and hence there is an involution in $G^{\circ}$ swapping two generic and independent elements of $\Omega$. Since $G^{\circ}$ is connected and contains an involution, it is not of degenerate type.

This really does require primitivity in addition to generic 2-transitivity.

Example 9. Let $G=G_{0} \times G_{1}$, where $G_{i}=\mathrm{GL}\left(V_{i}\right)$ and $V_{i}$ are vector spaces over fields $K_{i}$, with $K_{0}$ of characteristic two and $K_{1}$ not of characteristic two. Then $G$ has mixed type and acts generically $n$-transitively on $V_{1} \times V_{2}$, where $n$ is the minimal dimension involved.

5.2. Even type groups. In the simple case, we can use the classification in even type to reduce the analysis to the specific case of Chevalley groups. The theory in odd type is not sufficiently advanced to allow this kind of sweeping reduction to known groups, though there is a large body of work also in this direction.

Lemma 5.4. Let $(G, \Omega)$ be a transitive group of finite Morley rank, where the group $G$ is isomorphic as an abstract group to a Chevalley group over an algebraically closed field. Then the algebraic dimension of a maximal torus $T$ of $G$ is at most $\operatorname{rk}(\Omega)$, and if the characteristic of the base field is positive then $\operatorname{rk}(T) \leq \operatorname{rk}(\Omega)$.

Proof. By Lemma 3.8, $\operatorname{rk}(T / O(T)) \leq \operatorname{rk}(\Omega)$. Now $T$ is definably a direct product of 1-dimensional tori $T_{i}$ and $O(T)=\prod O\left(T_{i}\right)$, so $T / O(T)$ is also a product $\prod T_{i} / O\left(T_{i}\right)$ and therefore $\operatorname{rk}(T / O(T))$ is at least the number of factors.

Now in the case of positive characteristic we also know that $T$ is a good torus by Wagner's results, and thus $O(T)=1$.

Proposition 5.5. Let $(G, \Omega)$ be a simple permutation group of finite Morley rank and even type. Then the rank of $G$ is bounded by a function of the rank of $\Omega$ (e.g., $\left.4 \operatorname{rk}(\Omega)^{2}\right)$.

Proof. Due to simplicity of $G$, we can assume without loss of generality that $G$ is transitive on $\Omega$. If $d$ is the algebraic dimension of $G$ and $\ell$ the Lie rank, then for the classical groups of types $A_{\ell}, B_{\ell}, C_{\ell}, D_{\ell}$ we have $d=\ell(\ell+2)$ or $\ell(2 \ell \pm 1)$, so at most $2 \ell^{2}+\ell$. This leaves aside only the exceptional groups of types $E_{6}-E_{8}, F_{4}, G_{2}$, for which the Lie ranks and dimensions are: $(6,78) ;(7,133) ;(8,248) ;(4,52) ;(2,14)$ respectively. 
Let $T$ be a maximal torus in $G$. Now if $f$ is the rank of the base field (visible in various ways in the group, notably via the root groups), then $\operatorname{rk}(G)=d f$ and $\operatorname{rk}(T)=\ell f$. So it suffices to bound $d / \ell$ in terms of $\operatorname{rk}(\Omega)$, and since $\ell \leq \operatorname{rk}(T) \leq$ $\operatorname{rk}(\Omega)$ by Lemma 5.4 , this is certainly possible.

So in view of Lemma 5.3 the problem now is to get some control over actions of simple groups of odd type without an explicit classification (and even for Chevalley groups in characteristic zero there is something still to analyze).

5.3. Odd type groups. We now need some general structural properties of odd type groups, and we have to elaborate a little on the existing theory. Our starting point is the following.

Definition 5.6. A decent torus is a divisible abelian group of finite Morley rank which is the definable hull of its torsion subgroup.

Theorem 11 ([9]). Let $T$ be maximal definable decent torus in a connected group $G$ of finite Morley rank. Then the generic element of $G$ belongs to a unique conjugate of $C^{\circ}(T)$. Furthermore, any two maximal decent tori of $G$ are conjugate.

The following inessential variant can be proved the same way, or simply deduced from the foregoing.

Proposition 5.7. Let $G$ be a connected group of finite Morley rank and $T$ a maximal p-torus of $G$. Then the generic element of $G$ lies in one and only one conjugate of $C^{\circ}(T)$. Furthermore, any two maximal p-tori of $G$ are conjugate in $G$.

Proof. Since any maximal $p$-tori extend to maximal decent tori, the conjugacy statement here follows from the preceding.

Now let $\hat{T}$ be a maximal decent torus containing the given $p$-torus $T$. Then for a generic set of elements $a \in G, a$ lies in one and only one conjugate of $C^{\circ}(\hat{T})$, and in particular a centralizes a conjugate of $T$. We may suppose $a$ centralizes $\hat{T}$.

If $a$ centralizes the maximal $p$-torus $T_{1}$ of $G$, and $\hat{T}_{1}$ is a maximal decent torus in $C^{\circ}(a)$ containing $T_{1}$, then $T$ and $T_{1}$ are conjugate in $C^{\circ}(a)$ and hence $\hat{T}_{1}$ is also a maximal decent torus of $G$. Thus $\hat{T}_{1}=\hat{T}$ (by our choice of $a$ ) and $T_{1} \leq \hat{T}$. So by maximality of $T$ and $T_{1}$, we have $T=T_{1}$.

In a similar vein the following will be useful.

Lemma 5.8. Let $G$ be a connected group of finite Morley rank containing no nontrivial unipotent $p$-subgroup. Then for a generic element a of $G$, the group $d(a)$ is p-divisible.

Proof. Let $T$ be a maximal $p$-torus of $G$. Let $X \subseteq G$ be the set of elements belonging to a unique conjugate of $C^{\circ}(T)$. This set is generic in $G$ and disjointly covered by conjugates of $X \cap C^{\circ}(T)$. So it suffices to look at generic elements of $C^{\circ}(T)$. In other words, we may suppose that the maximal $p$-torus $T$ is central in $G$.

We claim then that all elements of order $p$ in $G$ belong to $T$. Let $\hat{T}=d(T)$ be the definable hull of $T$, a divisible abelian group whose $p$-torsion lies in $T$. Our claim is that $G / \hat{T}$ contains no $p$-torsion. Otherwise, $\bar{G}=G / \hat{T}$ contains a nontrivial connected definable abelian $p$-subgroup $\bar{K}_{0}$, whose definable hull is the image of a connected nilpotent subgroup $K$ of $G$. Now $K$ is a connected nilpotent group 
containing $p$-torsion not in $T$, and as $T$ is a maximal $p$-torus in $K$ there must be a nontrivial $p$-unipotent subgroup in $K$, contradicting our hypothesis.

Now consider $a \in G$ generic over $T$ (which is a countable set). Then $d(a)$ has the form $A \times T_{0}$ with $A p$-divisible and $T_{0}$ a finite cyclic $p$-group. In particular $T_{0} \leq T$.

Now $a=b t$ with $b \in A$ and $t \in T_{0}$. So $A T_{0}=d(a) \leq d(b) \times\langle t\rangle$ and thus $A=d(b)$, $T_{0}=\langle t\rangle$. But $b=a t^{-1}$ is also generic over $T$, and $d(b)$ is $p$-divisible. As there is a unique generic type in $G$, we must have $d(g) p$-divisible for any realization $g$ of this type.

Unlike most results of this general character, the lemma does not yield a generic definable set of elements $g$ for which the definable hull $d(g)$ is $p$-divisible. For example, working in an ordinary algebraic torus over an algebraically closed field, any generic set will contain $p$-elements for all primes $p$ different from the characteristic, and for such elements the definable hull is the finite cyclic group generated by them.

The following specialized result will be quite useful for the analysis of groups of odd type as permutation groups.

Proposition 5.9. Let $G$ be a connected group of finite Morley rank and odd type, and let $T$ be a maximal 2-torus of $G$. Then $T$ contains all the involutions in $C(T)$.

Proof. It is convenient to generalize this a little, allowing $G$ also to be of degenerate type (i.e. $T=1$ ), in which case we arrive at the nontrivial but known result that degenerate type groups contain no involutions.

We suppose toward a contradiction that $G$ is a minimal counterexample, and that $i \in G$ is an involution centralizing a maximal torus $T$ of $G$, with $i \notin T$. We make a number of reductions. Note that $i$ cannot belong to any proper definable connected subgroup containing $T$. Furthermore $G$ is nonabelian.

$$
Z^{\circ}(G)=1
$$

The connected abelian subgroup $Z^{\circ}(G) d(T)$ does not contain $i$. Therefore in the quotient $\bar{G}=G / Z^{\circ}(G)$, the subgroup $\bar{T}$ is a maximal 2-torus centralizes by an involution $\bar{i}$ which lies outside $\bar{T}$. If $Z^{\circ}(G)>1$ then we contradict the minimality of $G$ (or $T \leq Z^{\circ}(G)$ and then we reduce to the degenerate case). So $Z^{\circ}(G)=1$.

$$
\text { Without loss of generality, } Z(G)=1
$$

What we actually have is $Z(G)$ finite, and we wish to factor out the center. For this it suffices to check first that $i \notin Z(G) T$. Assuming the contrary, we may even take $i \in Z(G)$.

Now a generic element of $G$ lies in a conjugate of $C^{\circ}(T)$. So for a generic set of elements $g \in G$, both $g$ and $g i$ lie in subgroups of the form $C^{\circ}\left(T_{1}\right), C^{\circ}\left(T_{2}\right)$ for some conjugates $T_{1}, T_{2}$ of $T$. In particular $T_{1}, T_{2} \leq C^{\circ}(g)$. So $T_{1}$ and $T_{2}$ are conjugate in $C^{\circ}(g)$. Since $g \in C^{\circ}\left(T_{1}\right)$, we find $g \in C^{\circ}\left(T_{2}\right)$ as well. Since $g i \in C^{\circ}\left(T_{2}\right)$ we find $i \in C^{\circ}\left(T_{2}\right)$. So conjugating in $G$, we find $i \in C^{\circ}(T)$ as well. Now $C^{\circ}(T) / d(T)$ has degenerate type and hence contains no involutions. That is, $i \in d(T)$ and thus $i \in T$, a contradiction. Replacing $G$ by $\bar{G}=G / Z(G)$, we may suppose $Z(G)=1$.

As $C^{\circ}(i)<G$, minimality of $G$ yields

$$
i \notin C^{\circ}(i)
$$


This is the sort of pathological situation in which a genericity argument becomes available.

We consider the coset $i C^{\circ}(i)$. A generic element of this coset has the form $i a$ with $a$ a generic element of $C^{\circ}(i)$ (treating $i$ as a fixed parameter), and in particular there is one and only one conjugate $T_{a}$ of $T$ in $C^{\circ}(i)$ centralizing $a$.

Now $d(a)$ is 2-divisible by Lemma 5.8 , and $\left[d(a): d\left(a^{2}\right)\right] \leq 2$, so $d(a)=d\left(a^{2}\right)$ and thus $a \in d(a i)$ and $d(a i)=d(a, i)$. Hence $C(a i)=C(a) \cap C(i)$ and thus $T_{a}$ is the is also the unique conjugate of $T$ in $G$ commuting with ai.

Let $X=\left\{a C^{\circ}(i): a\right.$ is generic in $C^{\circ}(i)$ over $\left.i\right\}$. Suppose that $x \in X \cap X^{g}$ for some $g \in G$, so that

$$
x=a i=(b i)^{g}
$$

with $a$ and $b$ generic over $i$ in $C^{\circ}(i)$. Then $T_{a}=T_{a i}$ and $T_{b}=T_{b i}$. For notational simplicity suppose that $T_{a}=T$. Then $T_{a}=T_{b}^{g}$. There is also some $h \in C^{\circ}(i)$ with $T^{h}=T_{b}$. Then $T^{h g}=T$ and $h g \in N(T)$.

Furthermore we have $i^{g}=i^{h g} \in C(T) \cap i C^{\circ}(i)$, since $i^{g} \in d(b i)^{g}=d(a i)$. Writing $i^{g}=i j$ with $j \in C^{\circ}(i)$, we see that $j \in C^{\circ}(i)$ commutes with $T$, so by induction $j \in T$. Thus $i \in i \Omega_{1}(T)$. This is a finite set.

Now suppose the element $x$ lies in $X^{g_{1}} \cap X^{g_{2}}$ and the conjugates $i^{g_{1}}, i^{g_{2}}$ are equal. Then $g_{1} g_{2}^{-1} \in C(i)$, and therefore $X^{g_{1}}=X^{g_{2}}$. So all of this shows that $x$ belongs to only finitely many distinct conjugates of $X$. Let the number of such conjugates be $k$.

The type $X$ (over $i$ ) does not have $k+1$ distinct conjugates which intersect. So the same applies, by compactness, to some $i$-definable subset $\hat{X}$ of $i C^{\circ}(i)$ containing $X$. We claim that $\bigcup \hat{X}$ is generic in $G$.

We have $N(\hat{X}) \leq N\left(C^{\circ}(i)\langle i\rangle\right)$ since $\hat{X}$ generates $C^{\circ}(i)\langle i\rangle$. So by a Frattini argument we have $N(\hat{X}) \leq C(i) N(T\langle i\rangle)$. Now $N^{\circ}(T\langle i\rangle)=C^{\circ}(T\langle i\rangle) \leq C(i)$, so $N^{\circ}(\hat{X})=C^{\circ}(i)$. Now a standard rank computation (first used in the theory of bad groups, and much used recently, cf. [13]), shows that the union $\bigcup \hat{X}^{G}$ is generic in $G$.

However for all $x \in \hat{X}$, the group $d(x)$ is not 2-divisible, while for generic $g \in G$, the group $d(g)$ is 2-divisible, and we arrive at a contradiction.

We tidy this up a little more.

Corollary 5.10. Let $G$ be a connected group of finite Morley rank and odd type, and let $T$ be a maximal 2-torus of $G$. Then $T$ contains all the 2-elements in $C(T)$.

Proof. Supposing on the contrary that $t$ is a 2-element in $C(T)$ outside $T$, we may take $t$ to have order 2 modulo $T$. Then taking $t_{0} \in T$ with $t_{0}^{2}=t^{2}$ we have $t t_{0}^{-1} \in T$ by the previous proposition.

5.4. Primitive simple groups of odd type. Now we come back to permutation groups. We are interested in working with the action of a maximal 2-torus in a definably primitive simple permutation group of odd type.

Lemma 5.11. Let $(G, \Omega)$ be a definably primitive permutation group of finite Morley rank with $G$ of odd type, and let $r=\operatorname{rk}(\Omega)$. Then for any $k$ there is an $N \leq k r+1$ such that for any sequence

$$
x_{1}, \ldots, x_{N}, \ldots, x_{N+k}
$$


of independent generic elements of $\Omega$, the point stabilizer $G_{N+k}=G_{x_{1}, \ldots, x_{N+k}}$ contains a maximal 2-torus of $G_{N}=G_{x_{1}, \ldots, x_{N}}$.

Proof. Take an infinite sequence $x_{1}, x_{2}, \ldots$ of independent generic elements of $\Omega$. Let $G_{i}$ be the point stabilizer $G_{x_{1}, \ldots, x_{i}}$. Let $T_{i}$ be the definable hull of a maximal 2-torus of $G_{i}$, and let $r_{i}=\operatorname{rk}\left(T_{i} / O\left(T_{i}\right)\right)$.

The sequence $r_{i}$ is monotonically decreasing and takes on at most $r+1$ values in view of Lemma 3.8. Thus over the interval $[1, \ldots, k(r+1)+1]$ there must be an interval $[N, N+k]$ of length $k$ over which the function is constant. Here $N \leq k r+1$.

Suppose now that $T_{1}$ is the definable hull of a maximal 2-torus of $G_{N+k}$ and that $T_{2}$ is the definable hull of a maximal 2-torus of $G_{N}$ with $T_{1} \leq T_{2}$. As $r_{N}=r_{N+k}$ we have

$$
\operatorname{rk}\left(T_{1} / O\left(T_{1}\right)\right)=\operatorname{rk}\left(T_{2} / O\left(T_{2}\right)\right)
$$

Thus $T_{2}=T_{1} O\left(T_{2}\right)$ and hence the maximal 2-torus of $T_{2}$ is contained in $T_{1}$, as claimed.

Proposition 5.12. Let $(G, \Omega)$ be a definably primitive permutation group of $f$ nite Morley rank with $G$ connected and of odd type. Then the degree of generic transitivity of $G$ is at most $4 r^{2}+5 r+1$ for $r=\operatorname{rk}(\Omega)$.

Proof. Take an infinite sequence $x_{1}, x_{2}, \ldots$ of independent generic elements of $\Omega$. Let $k=4 r+4$. Suppose that $G$ is generically $[k(r+1)+1]$-transitive. Apply the preceding lemma to get a corresponding value of $N$. Let $H$ be the point stabilizer $G_{x_{1}, \ldots, x_{N}}$ and Let $F$ be the set

$$
\left\{x_{N+1}, \ldots, x_{N+k}\right\}
$$

Then $H_{F}$ is the point stabilizer $H_{x_{N+1}, \ldots, x_{N+k}}$. As $G$ is generically $(N+k)$-transitive, the setwise stabilizer $H_{\{F\}}$ induces the full symmetric group $\operatorname{Sym}(k)$ on $F$.

Fix a maximal 2-torus $T_{0}$ of $H_{F}$ and let $T$ be its definable hull. By the Frattini argument there is a subgroup of $H_{\{F\}}$ normalizing $T$ and covering $\operatorname{Sym}(k)$. Modulo the kernel of the action on $T / O(T)$, the group still covers $\operatorname{Sym}(k)$, as its 2-elements are forced to act nontrivially on $T_{0}$ by Corollary 5.10 .

This then yields $\operatorname{rk}(T / O(T)) \geq\lfloor k / 4\rfloor>r$ by Proposition 4.1, violating our estimate in Lemma 3.8.

Note that for $r=2$ this gives a bound on generic transitivity of at most 27 for a definably primitive action. Everything depends on the bound in Proposition 4.1 and there is considerable room for improvement here, particularly when $r$ is low.

The bound in [11] for sharp generic transitivity without assuming definable primitivity is 6 . In the actual example of $\mathrm{SL}(n)$, with generic sharp $(n+1)$-transitivity, a maximal torus of rank $n-1$ appears in the stabilizer of $n$ independent points with a group $\operatorname{Sym}(n)$ acting on it, so the various estimates do not give up too much up to this point. But after fixing another generic independent point one loses the whole torus in this case. It would also be helpful to understand whether double covers of $\operatorname{Sym}(n)$ can occur in the normalizer of a maximal 2-torus.

5.5. Tying up the loose ends. Finally, we have to assemble a proof of Theorem 1 from several pieces developed in the paper.

Notice, first of all, that results of Section 3 reduce Theorem 1 to its special case for simple groups $G$, Theorem 2 . 
To get a bound for $\operatorname{rk}(G)$ in Theorem 2, it will suffice, in view of Proposition 2.2, to bound the generic transitivity degree of a simple group $G$ in terms of the rank of the set on which it acts; this intermediate result is formulated as Proposition 3.1.

In a proof of Proposition 3.1, we may assume that $G$ has sufficiently high generic transitivity degree; in particular, $G$ is generically 2 -transitive, hence contains involutions and is therefore a group of odd or even type (Lemma 5.3). The bound in the even case is provided by Proposition 5.5; the odd case is dealt with in Proposition 5.12 .

This completes the proof of Theorem 1 .

\section{Generic multiple transitivity}

6.1. An extremal case. The following conjecture has the potential to involve a good deal of the existing theory of groups of finite Morley rank.

Problem 9. Let $G$ be a connected group of finite Morley rank acting faithfully, definably, transitively and generically $(n+2)$-transitively on a set $\Omega$ of Morley rank $n$. Then the pair $(G, \Omega)$ is equivalent to the projective linear group $\mathrm{PGL}_{n+1}(F)$ acting on the projective space $\mathbb{P}^{n}(F)$ for some algebraically closed field $F$.

Of course the transitivity hypothesis is superfluous as one may restrict to the relevant orbit in any case. It is here just to keep the notation clean. Note that we aim to show in particular that the action of $G$ is primitive.

This conjecture would give natural bounds for generic multiple transitivity of permutation groups of finite Morley rank. But the main point is that it provides a convenient sandbox for trying out recently developed methods, in particular those used in the ongoing analysis of groups of odd type. A major part of this conjecture is the "affine group" case which might come in to the analysis of the point stabilizer of the group from Problem 9; see Problem 13 below.

One reason that induction may be useful is the following.

Lemma 6.1. Let $(G, \Omega)$ be a transitive and generically $n$-transitive permutation group of finite Morley rank. Let $E$ be a definable $G$-invariant equivalence relation on $\Omega$. Then $(G, \Omega / E)$ is generically $n$-transitive (though not necessarily faithful).

Proof. As $G$ acts transitively on $\Omega / E$, the equivalence classes have constant rank. So generic sets in $\Omega$ and $\Omega / E$ correspond.

Taking an inductive approach to Problem 9, then under the stated hypotheses it follows that any proper $G$-invariant definable equivalence relation $E$ will have finite classes. One can then show that there is a maximal such relation $E$, definable by $E(\alpha, \beta) \Longleftrightarrow G_{\alpha}{ }^{\circ}=G_{\beta}{ }^{\circ}$. There is a potential issue with the uniform definability of $G_{\alpha}{ }^{\circ}$ but that is not a problem for transitive actions. Modulo this maximal $E$, we have a definably primitive action. So if we have the desired result for definably primitive actions, then the pair $(G / K, \Omega / E)$ is identified, with $K$ the kernel of the action on the quotient. Now $K$ leaves each class of $E$ invariant, so $K^{\circ}$ acts trivially on $\Omega$, hence $K$ is finite and $G$ is a central extension of $G / K$. In particular $K$ is contained in a maximal torus $T$ of $G$. Now $T$ leaves some class $C$ of $E$ invariant, and being connected fixes $C$ pointwise. Hence $K$ has fixed points, and being central acts trivially on $\Omega$. So $K=1$. So without loss of generality one may suppose $(G, \Omega)$ is definably primitive in Problem 9. 
Thus we may bring [14] to bear again, and now the group $G$ is connected. This is a very special case, and the classification then reduces to the following possibilities.

(1) The affine case: $G=A \rtimes H$ with $H$ a point stabilizer acting generically $(n+1)$-transitively on an elementary abelian group of rank $n$.

(2) The socle is a simple group $L$ acting regularly on $\Omega$ and $G=L \rtimes H$ with $H$ a point stabilizer acting generically $(n+1)$-transitively on the simple group $L$ of rank $n$ as a group of automorphisms.

(3) The socle is a simple group $L$ acting nonregularly and $G$ lies between $L$ and $\operatorname{Aut}(L)$.

(4) Diagonal action: the socle is the product of two isomorphic simple groups $L_{1} \times L_{2} . \quad L_{1} \times L_{2} \leq G \leq \operatorname{Aut}\left(L_{1}\right) \times \operatorname{Aut}\left(L_{2}\right)$ and furthermore $G /\left(L_{1} \times\right.$ $\left.L_{2}\right) \leq \Delta(\operatorname{Out}(L))$ where $L \cong L_{1} \cong L_{2}$ and $\Delta(\operatorname{Out}(L))$ is the subgroup of $\operatorname{Out}\left(L_{1}\right) \times \operatorname{Out}\left(L_{2}\right)$ corresponding to the diagonal subgroup via an isomorphism of $L_{1}$ with $L_{2}$. In other words, if $\hat{G}$ is the subgroup of $\operatorname{Aut}\left(L_{1}\right) \times$ $\operatorname{Aut}\left(L_{2}\right)$ consisting of pairs $(\alpha, \beta)$ with $\alpha \equiv \beta \bmod \operatorname{Inn}(L)$, we want

$$
L_{1} \times L_{2} \leq G \leq \hat{G}
$$

The set $\Omega$ is defined by specifying the point stabilizer, which is the diagonal subgroup $\Delta(\operatorname{Aut}(L))$ (or its intersection with $G$ ).

In the third and fourth cases the condition of finiteness of Morley rank is extremely restrictive. If $L$ is a Chevalley group then any connected subgroup of $\operatorname{Aut}(L)$ containing $L$ will coincide with $L$ itself, while $\operatorname{Aut}(L)$ includes everything induced by a field automorphism.

In Problem 9 our target is type (3) and therefore one of the things one wants to do, presumably, is to remove the other possibilities. However another idea is to use induction and focus on the point stabilizer. Probably one should do both, but the second seems like the main avenue of attack.

Let us examine the basic case of type (4), where $G=L \times L$ and $L_{\alpha}=\Delta(L)$. This is equivalent to the natural action of $L^{\mathrm{op}} \times L$ on $L$ on the left and right, which as an action of $L \times L$ becomes $(g, h) \cdot a=g^{-1} a h$. Thus $G_{1}=\Delta(L)$ and the action of $G_{1}$ on $L$ is equivalent to the action of $L$ on $L$ by conjugation.

The general case of (4) is similar. The set $\Omega$ can be identified with $\Delta(L) \backslash L \times$ $L$ which has as representatives either copy of $L$, and after this identification $G_{1}$ becomes a subgroup of $\operatorname{Aut}(L)$ containing $L$ and acting on $\Omega$ as it acts on $L$.

Now in both cases (2) and (4) there is a definable simple normal subgroup $L$ acting regularly on $\Omega$, on which the point stabilizer $G_{\alpha}$ acts as a group of automorphisms. Thus in these cases $\operatorname{rk}(L)=n$ and $G_{\alpha}$ acts generically $(n+1)$-transitively on $L$. By the proof of Lemma 3.3 if $a_{1}, \ldots, a_{n}$ are $n$ independent generic elements of $L$, then $d\left(a_{1}, \ldots, a_{n}\right)=L$ unless $\operatorname{rk}\left(d\left(a_{1}, \ldots, a_{\ell}\right)\right) \leq \ell-1$ for all $\ell \leq n$. In particular the generic element of $L$ has finite order.

Problem 10. Let $L$ be a connected $\aleph_{0}$-saturated group of finite Morley rank such that for two independent generic elements $a, b$ we have $\operatorname{rk}(d(a, b)) \leq 1$. Show that $L$ is nilpotent of bounded exponent.

This, or something similar, would get rid of these two cases for our present problem.

Alternatively, as we will see later, in many such cases one can force the socle to act generically doubly transitively and in particular get an involution into $L$, in which case it is definitely of unbounded exponent, as it is not of degenerate type. 
One may also use Lemma 3.8 to study the point stabilizer $M=G_{\alpha}$ on an appropriate definable quotient $\Omega / E$. Here one hopes to arrive at a configuration where modulo the kernel $K$ of the action, $M / K$ contains a subgroup $\operatorname{PGL}_{n}(F)$, while $K$ has Prüfer rank at most 1 . This configuration deserves close study. In particular, one should try to deal with the following.

Problem 11. If a simple algebraic group $L$ acts definably on a group $K$ of degenerate type, then $[K, L]$ is nilpotent.

There is a similar conjecture for actions of the definable hull of a 2-torus on a degenerate type group, which would give the solution to this problem as a special case. But perhaps this version is more accessible.

6.2. The affine case. For the affine case, we want the following.

Problem 12. Let $G$ be a connected group of finite Morley rank acting faithfully, definably, and generically t-transitively on an abelian group $V$ of Morley rank $n$. Then $t \leq n$.

Later, with an eye on the point stabilizer, we may also want the extremal affine case treated fully. Whether or not it enters directly into the treatment of Problem 9 , it is of independent interest.

Problem 13. Let $G$ be a connected group of finite Morley rank acting faithfully, definably, and generically $n$-transitively on a connected abelian group $V$ of Morley rank $n$. Then $V$ has a structure of a $n$-dimensional vector space over an algebraically closed field $F$ of Morley rank 1 , and $G$ is $\mathrm{GL}_{n}(F)$ in its natural action on $F^{n}$.

This problem connects closely to the structure theory for groups of finite Morley rank. Note that Problem 12 is a special case of Problem 13.

In either of these problems $A$ should be $G$-minimal, if we are in an inductive setting. We have only to consider the possibility of a nontrivial $G$-invariant finite subgroup $A_{0}$, in which case $G$ fixes $A_{0}$ pointwise. Passing to $A / A_{0}$ we either have a contradiction or an identification of $G / K$ acting on $A / A_{0}$ with $\mathrm{GL}_{n}(F)$ in its natural action, where $K=C_{G}\left(A_{0}\right)$. As usual $K^{\circ}$ acts trivially $A$ and $K$ is finite. As $G$ is connected we find $K=1$. So now we have

$$
1 \rightarrow A_{0} \rightarrow A \rightarrow A / A_{0} \rightarrow 1
$$

a sequence of $G$-modules with $A_{0}$ finite and trivial, and $A / A_{0}$ natural. The associated 2-cocycle with values in $A_{0}$ is "generically constant" in each variable, the extension so should split definably, another point to be checked. As $A$ is connected we have finally $A_{0}=1$.

In particular $A$ is either torsion free or an elementary abelian $p$-group for some $p$. In the context of Problem 12 only the elementary abelian case is possible, and in the context of Problem 13 one may have the torsion free case, with each subgroup of the form $d(a)$ of rank 1. But in the latter case one has also that the definable closure of $k$ independent generic elements has rank $k$ and that its setwise stabilizer acts generically $k$-transitively. This seems like enough to complete the analysis of the torsion free case in the full generality of Problem 13, by direct induction (perhaps by characterizing the underlying projective geometry, or by more group theoretic methods). Some particular arguments of a more concrete character may also be needed in low rank cases. 
Now return to Problem 12 in the case in which $A$ is an elementary abelian $p$-group.

Consider any nontrivial definable $G$-invariant equivalence relation $E$ such that a generic element of $A$ belongs to an infinite class. Then $G$ acts generically $t$ transitively on the quotient of the generic orbit modulo $E$ and if $t>n$ this forces the ranks of the generic classes to be 1 , by induction on $n$, and then the action on the quotient and the induced group $G / K$ can be identified with $(\operatorname{PGL}(n-$ $\left.1, F), \mathbb{P}^{n-1}(F)\right)$. In particular the setwise stabilizer of a single generic $E$-class $C$ is its pointwise stabilizer in this representation, and acts on the class $C$, which however has rank 1. It follows that the whole class $C$ has a nontrivial pointwise stabilizer $G_{C}$ in $G$. Let $\hat{C}$ be the fixed point set of $G_{C}$. This is a proper subgroup of $A$. One can recover $\hat{C}^{\circ}$ from its generic element. So this gives rise to another $G$-invariant equivalence relation on the generic orbit in $A$, again with classes of rank 1 , but now each class is generic in the group it generates. So we cover $A$ by a collection of $G$-invariant subgroups with finite intersections. Actually the intersection of each subgroup with the union of its distinct conjugates is finite and $G$-invariant so by $G$-minimality these intersections are trivial.

Now it seems the kernel $K$ of the quotient action is acting as an abelian subgroup on each class $C$ (group $C \cup\{0\}$ ) and hence is itself abelian. And also a good torus. So central in $G$. Hence semi-regular. So if this is nontrivial then we have just put a linear structure on $A$ and that is the end of generic $(n+1)$-transitivity. So $K$ is finite and $G$ is a covering group of $\operatorname{PGL}(n-1, F)$. But this acts generically sharply $n$-transitively on the quotient so after fixing $n$ generic independent points in $A$ we are out of group elements.

All of which says that as one expects any $G$-invariant equivalence relation has generically finite classes. And for such a class $C$ and $a \in C, C$ is contained in the fixed point set for $G_{a}{ }^{\circ}$, which is a finite subgroup of $A(a \neq 0)$. So we have again a primitive quotient of $(G, A)$ or rather $(G, O)$ with $O$ the generic orbit, and there is at worst a finite kernel. So $G$ is itself subject to MPOSA. An infinite abelian normal subgroup in $G$ would linearize $A$ again and lead to a contradiction, so the socle of $G$ is again simple or a product of two simple groups; actually quasisimple allowing for the finite kernel.

Two simple groups will lead again to linearization and a contradiction so the socle is a single simple subgroup $L$. As $L$ acts transitively on the primitive quotient of the generic orbit it also acts transitively on that orbit.

Suppose $A$ contains a nontrivial infinite definable proper subgroup. Then fixing a minimal one $A_{0}, A$ is more or less a direct sum of conjugates of $A_{0}$ by finitely many elements of $L$. Then the generic element of $A$ is a sum of independent generic elements in these conjugates (which can all be identified with $A_{0}$ ). So the generic $(n+1)$-transitivity of $G$ on $A$ gives the same thing on $A_{0}$, in lower rank, and a contradiction. So $A$ is minimal in this strong sense.

Now $G$ contains involutions since it is generically doubly transitive. An involution has finite centralizer on $A$ so inverts $A$. But we have a 2-element interchanging generic independent elements so an involution swaps them or fixes them, a contradiction.

So it seems that in Problem 9 our original group $G$ must have a simple socle $L$ acting nonregularly. 
If this socle is of even type then it is a Chevalley group, and $G=L$, and one is in the algebraic case, more or less. Actually as the language is enriched there are potentially more permutation representations than in the algebraic category. For the truly algebraic case generically multiply transitive actions of simple groups (also reductive groups) have been studied by Popov [19] by a method which is limited to characteristic zero for technical reasons. One does not get beyond generic 4transitivity except in the case of the series $A_{\ell}$, as anticipated.

Problem 14. Extend Popov's work to all characteristics and to the finite Morley rank permutation group category in which the groups are Chevalley groups, or products of Chevalley groups and tori.

In our category the base field can vary from group to group in a direct product, and it is not clear what a "torus" actually is, but good tori would be a natural candidate for the role. In any case it is the base case of Chevalley groups which is critical here.

This approach also leaves low values of $n$ to deal with. Passing through this kind of classification is cumbersome for low values of $n$ and one should look for more intrinsic methods.

The basis of the induction is discussed again, more generally, in Problems 15 and 16 formulated below.

If the even type case is eliminated then while $G$ is of odd type, the socle of $G$ may, in principle, be of either degenerate or odd type. It is not clear how to limit things further on an a priori basis, so it would seem to be high time to take up the point stabilizer, which also has a generically highly transitive action.

Note incidentally that we may assume

The point stabilizer is connected.

This amounts to replacing $\Omega$ by a finite cover where the point stabilizer is replaced by its connected component.

If one succeeds in identifying the point stabilizer (and the ambient group) after this change, then all that remains is to check that it is self-normalizing in the larger group.

6.3. The point stabilizer. In the context of Problem 9 it would be nice to show that the point stabilizer has a faithful primitive permutation representation, and to argue that it falls in the affine class, then identify the affine action explicitly as the action of $\mathrm{GL}(n)$ on its natural module. After which one would still have to identify the original group, and the whole of the point stabilizer. However it is not so clear how to extract an appropriate representation from the data.

Let us consider the action of the point stabilizer $G_{\alpha}$ on $\Omega$. This may be definably imprimitive. In that case one gets equivalence classes of rank 1 and a quotient of $G_{\alpha}$ to which induction applies. The connected component of the kernel operates on a collection of rank 1 sets and is therefore either solvable or has factors of type PSL(2). So one has, in this case, a great deal of information about $G_{\alpha}$ and one may hope to disentangle the situation from this point.

On the other hand the action of $G_{\alpha}$ on its generic orbit may be definably primitive, and while this again restricts the structure of $G_{\alpha}$, it is not so strong, and needs to be driven toward an eventual contradiction. In particular $G_{\alpha}$ may have 
simple socle, in which case we are forced again to deal with simple groups having an unclear structure.

However, if $L$ is the simple socle of $G$, then $L_{\alpha}$ contains the socle of $G_{\alpha}$ and by primitivity acts transitively on the generic orbit of $G_{\alpha}$. So in this case at least $L$ acts generically doubly transitively and in particular contains involutions, so is of odd type.

6.4. Small affine groups. Returning now to the affine case, we comment on the case of low rank, which would seem to require separate treatment.

Problem 15. Let $G$ be a connected group of finite Morley rank acting faithfully and definably on an abelian group $V$ of Morley rank 2. Then either $G$ is solvable, or $V$ has a structure of a 2-dimensional vector space over an algebraically closed field $F$ and $G$ is one of the groups $\mathrm{SL}_{2}(F)$ and $\mathrm{GL}_{2}(F)$ in their natural action on $V=F^{2}$.

Problem 15 is believed to be solved, though all such claims must be checked.

Problem 16. Let $G$ be a connected group of finite Morley rank acting faithfully, definably and irreducibly on an abelian group $V$ of Morley rank 3 . Then one of the following holds:

- $V$ is of finite exponent $p>2$ and $G$ is a simple p-group.

- $V$ has a structure of a 3-dimensional vector space over an algebraically closed field $F$ and $G=Z L$ where $Z$ is a subgroup of the group of scalar matrices and $L$ is one of the groups $\mathrm{PSL}_{2}(F)$ (in its irreducible representation as a 3-dimensional orthogonal group) or $\mathrm{SL}_{3}(F)$ (in its natural action on $\left.F^{3}\right)$.

For the treatment of Problem 16, one would have to collate all known information on "small" groups of odd type, including Altseimer's work on characterization of $\mathrm{PSL}_{3}$ by centralizers of involutions. When $G$ happens to be $\operatorname{PSL}_{2}(F)$, turning $V$ into a vector space over $F$ could be a problem (unless this case is already covered by Meierfrankenfeld [16] — but we have not checked the details).

The $\mathrm{PSL}_{2}(F)$ case of Problem 16 is likely to come out of the configuration which appears to be covered by the recent theorem by Adrien Delore [10] on minimal simple groups of Prüfer rank 1.

Fact 6.2. Let $G$ be a minimal simple group of odd type and Prüfer rank 1. Assume that the centralizer of a toric involution is not a Borel subgroup in $G$. Then $G \simeq$ $\mathrm{PSL}_{2}(F)$ for an algebraically closed field $F$.

Eric Jaligot has informed us that the proof of Delore's theorem as such uses the solvability assumption only for "local" subgroups in $G$ : that, subgroups of the form $N_{G}^{\circ}(A)$ for an abelian subgroup $A<G$. Exactly this condition, solvability of local subgroups, will naturally appear in the treatment of Problem 16. It would be nice if someone would undertake the task of checking that all papers on minimal simple groups actually use only this weaker assumption; this would make Fact 6.2 immediately applicable in the analysis of Problem 16.

The following fact could be useful in proofs of Problems 15 and 16. (Again, the proof of this "fact" is being written up.)

Fact 6.3. Let $G$ be a simple group of finite Morley rank acting faithfully and definably on an elementary abelian p-group $V$. If $G$ contains no infinite elementary abelian p-subgroups then the following statements hold. 
- Every connected solvable subgroup of $G$ is abelian.

- If $C$ is a Carter subgroup of $G$ then $C_{G}(C)$ is generous in $G$.

- Carter subgroups of $G$ are conjugate.

- $G$ contains no involutions.

In the course of the proof of Fact 6.3 the following observation arises; one would expect it to be very useful in the proof of Problem 16.

Fact 6.4. Let $H$ be a connected group of finite Morley rank acting faithfully and definably on an elementary abelian p-group $V$. Assume that $H=L T$ where $L \triangleleft H$ and $T$ is an divisible abelian group equal to the definable closure of its 2-torsion. If $L$ contains no infinite elementary abelian p-subgroups then $[L, T]=1$.

6.5. An inductive step for Problem 13. The inductive step in the treatment of Problem 13 also appears to be natural: we take a generic point $v \in V$ and consider

$$
U=C_{V}\left(C_{G}(v)\right)
$$

the aim is to show that $\operatorname{rk}(U)=1$ and that therefore $H=N_{G}^{\circ}(U) / U$ acts generically $(n-1)$-transitively on $V / U$, which would allow us to apply the inductive assumption and conclude that $\bar{H}=H / C_{H}(V / U)$ is $\mathrm{GL}_{n-1}(F)$.

The next step is to glue together a Curtis-Tits system in a big subgroup $G^{*}$ of $G$ from a Curtis-Tits system in $[\bar{H}, \bar{H}] \simeq \mathrm{SL}_{n-1}(F)$ and from a Curtis-Tits system in a similar section of an appropriate conjugate of $H$. We discuss Curtis-Tits systems in the next subsection.

Finally, if a Curtis-Tits system in $G^{*}$ is constructed and $G^{*}$ is identified with $\mathrm{GL}_{n}(F)$ for an algebraically closed field $F$, the structure of an $F$-vector space on $V$ is introduced by Meierfrankenfeld's characterization of natural modules for classical groups [16].

6.6. The Curtis-Tits Theorem. We start by quoting a very general form of the Curtis-Tits Theorem in the formulation due to Timmesfeld [20].

Fact 6.5 ([20]). Let $\Phi$ be an irreducible spherical root system of Tits rank at least 3, with fundamental system $\Pi$ and Dynkin diagram $\Delta$. Let $G$ be any group generated by rank one groups $X_{r}=\left\langle A_{r}, A_{-r}\right\rangle$ for $r \in \Pi$, with unipotent subgroups $A_{r}, A_{-r}$ satisfying the condition

$$
N_{X_{r}}\left(A_{r}\right) \cap N_{X_{r}}\left(A_{-r}\right) \leq N\left(X_{s}\right)
$$

for all $r, s \in \Pi$. Set $X_{r s}=\left\langle X_{r}, X_{s}\right\rangle$ for $r, s \in \Pi$ distinct, and assume the following all hold.

(1) $X_{r}, X_{s}$ commute for $r, s$ not connected in $\Delta$.

(2) If $r, s$ are connected in $\Delta$, then there is a group $\bar{X}=\bar{X}_{r s}$ of Lie type with root system $\Phi_{r s}$ (the span of $r, s$ in $\left.\Phi\right)$, which is generated by subgroups $\bar{A}_{\alpha}$ for $\alpha \in \Phi_{r, s}$, and there is a surjective homomorphism $\phi_{r s}: X_{r s} \rightarrow \bar{X}_{r s}$, such that:

(a) $\phi_{r s}\left[A_{\alpha}\right]=\bar{A}_{\alpha}$ for $\alpha \in \Phi_{r s}$;

(b) $\operatorname{ker} \phi_{r s} \leq Z\left(X_{r s}\right)$;

(c) If $\bar{X}_{r s}$ is defined over a field of order 2 or 3 , or is of the form $\mathrm{PSL}_{3}(4)$, then $\operatorname{ker} \phi_{\text {rs }}$ is a $2^{\prime}$-group or a $3^{\prime}$-group respectively. 
A. BOROVIK AND G. CHERLIN

Then there is a group $\bar{G}$ of Lie type $\mathcal{B}$, with root system $\Phi$ and with fundamental system $\Pi$, and there is a surjective homomorphism $\sigma: G \rightarrow \bar{G}$ mapping the groups $A_{ \pm r}$ for $r \in \Pi$ onto the corresponding fundamental root groups and their opposites in $\bar{G}$. Furthermore, $\operatorname{ker} \sigma \leq Z(G) \cap H$, where $H$ is the subgroup generated by the groups $H_{r}=N_{X_{r}}\left(A_{r}\right) \cap N_{X_{r}}\left(A_{-r}\right)$ for $r \in \Pi$.

The following case is the one which concerns us here.

Proposition 6.6. Let $\Phi$ be an irreducible root system (of spherical type) and rank at least 3 , and let $\Pi$ be a system of fundamental roots for $\Phi$. Let $X$ a group generated by subgroups $X_{r}$ for $r \in \Pi$, Set $X_{r s}=\left\langle X_{r}, X_{s}\right\rangle$. Suppose that $X_{r s}$ is a group of Lie type $\Phi_{r s}$ over an infinite field, with $X_{r}$ and $X_{s}$ corresponding root $\mathrm{SL}_{2}$-subgroups with respect to some maximal torus of $X_{r s}$. Then $X / Z(X)$ is isomorphic to a group of Lie type via a map carrying the subgroups $X_{r}$ to root $\mathrm{SL}_{2}$-subgroups.

Since the only simple algebraic groups which we expect to appear in Problems 9 and 13 are groups $\mathrm{PSL}_{n}$ and $\mathrm{SL}_{n}$ over an algebraically closed field, we can specialize the Curtis-Tits Theorem even further.

The following fact is essentially Fact 5.2 of [4].

Proposition 6.7. Let $G$ be a group of finite Morley rank generated by a family of subgroups $K_{i}, i=1, \ldots, n-1, n \geqslant 3$. Assume that the following conditions hold:

- All $K_{i}$ are isomorphic to $\mathrm{SL}_{2}(F)$ for some algebraically closed field $F$ of characteristic $\neq 2$.

- $\left[Z\left(K_{i}\right), Z\left(K_{j}\right)\right]=1$ for all $i, j=1, \ldots, n-1$.

- $\left[K_{i}, K_{j}\right]=1$ if $|i-j|>1$.

- $\left\langle K_{i}, K_{j}\right\rangle \simeq \mathrm{SL}_{3}(F)$ if $|i-j|=1$.

Then $G$ is isomorphic to a factor group of the group $\mathrm{SL}_{n}(F)$ by a (finite) subgroup from the center.

Problem 16, which describes "3-dimensional" groups, could be very useful in the control of groups generated by two "root" $S L_{2}$-subgroups.

6.7. Pseudoreflection groups. Finally, Problem 13 may reduce to yet another Problem, also concerned with very familiar objects.

Problem 17. Let $G$ be a connected group acting definably, faithfully and irreducible on a abelian group $V$ (written additively). Assume that $G$ contains a pseudoreflection subgroup, that is, an abelian subgroup $R$ such that

(1) $V=[V, R] \oplus C_{V}(R)$

(2) $R$ acts transitively on the set of non-trivial elements in $[V, R]$.

Then $V$ has a structure of a vector space over an algebraically closed field $F$ such that $[V, R]$ is one-dimensional subspace and $R$ acts on $[V, R]$ as the multiplicative group of $F$, and $G=\mathrm{GL}(V)$.

It would be interesting to see whether the theory of pseudoreflection groups can be transferred from the case of groups of even type, where it features prominently in the classification theory of simple groups of finite Morley rank and even type, to the context of groups of odd type.

Notice that in the special case when we have $\operatorname{rk}([V, R])=1$, which is the only case needed for treating Problem 13, Problem 17 should follow easily from the results 
of Problems 15 and 16. However, at this point another theme comes into the plot: classical involutions.

Indeed, for any conjugate $R^{g}$ of $R$, the group $L=L_{g}=\left\langle R, R^{g}\right\rangle$ centralizes $U=C_{V}(R) \cap C_{V}\left(R^{g}\right)$ and acts on $V / U$. But $\operatorname{rk}(V / U) \leqslant 2$, and it will follow from Problem 15 that $\bar{L}=L / C_{L}(V / U)$ is isomorphic either to an abelian group $R * R^{g}$ (central product), or to a soluble group $F^{+} \rtimes F^{*}$, or to $\mathrm{GL}_{2}(F)$ for an algebraically closed field $F$, with $R$ and $R^{g}$ being one-dimensional algebraic tori.

The case in which all of the subgroups $\bar{L}_{g}$ are solvable, $g \in G$, is very peculiar and should lead to a contradiction with the assumption that $G$ acts on $V$ irreducibly.

Otherwise we choose $L$ such that $\bar{L} \simeq \mathrm{GL}_{2}(F)$. It should be easy to show that $C_{L}(V / U)=1$; after that, if we denote by $J$ the derived subgroup of $L \simeq \mathrm{GL}_{2}(F)$, we get a remarkable subgroup:

- $J \simeq \mathrm{SL}_{2}(F)$;

- if $z$ is the involution from $Z(J)$ then $J \triangleleft C_{G}(z)$;

- Let $J^{\prime}$ be a conjugate of $J$ and $z^{\prime}$ be the involution in $Z\left(J^{\prime}\right)$. If $z$ and $z^{\prime}$ commute then

$$
\left\langle J, J^{\prime}\right\rangle \simeq J \times J^{\prime}, J * J^{\prime} \text { or } \mathrm{SL}_{3}(F)
$$

(this is where Problem 16 would be useful).

The classical involution analysis in the spirit of Aschbacher and Berkman [4] should hopefully lead to the configuration of the Curtis-Tits Theorem (in the version of Proposition 6.7), and ultimately yield a subgroup $G^{*} \simeq \mathrm{SL}_{n}(F)$.

We can repeat a comment made in $\$ 6.5$ : if a Curtis-Tits system in $G^{*}$ is constructed and $G^{*}$ is identified with $\mathrm{SL}_{n}(F)$ for an algebraically closed field $F$, the structure of a $F$-vector space on $V$ is introduced by Meierfrankenfeld's characterization of natural modules for classical groups [16]. After that the identification of $G$ with $\mathrm{GL}(V)$ is likely to be very straightforward.

Notice also that Problem 17 could possibly provide a very efficient way to develop the Inductive Step in Problem 13: if, in the notation of $\S 6.5$, we can indeed conclude that $H / C_{H}(V / U)$ is $\mathrm{GL}_{n-1}(F)$, then a pseudoreflection subgroup from $\mathrm{GL}_{n-1}(F)$ in its action on $V / U$ is likely to induce a pseudoreflection subgroup action on $V$.

6.8. One more problem. The following arises in conjunction with attempts to analyze intersections of Carter subgroups in groups of degenerate type, which is a major focus of interest in the general theory, and may not be entirely out of place here.

Problem 18. Let $A$ be a connected abelian group of Morley rank $n$, and let $\left(A_{i}: i \in\right.$ I) be a uniformly definable family of pairwise distinct connected definable subgroups of rank $k$ in $A$. Show that $\operatorname{rk}(I) \leq\left(\begin{array}{l}n \\ k\end{array}\right)$.

The bound is achieved when $A$ is a vector space over a field of rank 1 . The case $n=2$ and $k=1$ is of particular interest.

\section{PROBLEM LIST}

We list here the problems that turned up along the way.

Problem 1 (Introduction). Bound (tightly, if possible) the rank of a definably primitive permutation group of finite Morley rank in terms of the rank of the set on which it acts. 
Problem $2(\S 1)$. If $(G, \Omega)$ is a permutation group and $\Omega$ is stable in the induced language, does it follow that $G$ is stable? Does this hold at least when $\Omega$ has finite Morley rank?

Problem $3(\S 1)$. Find all the generically sharply $n$-transitive actions of algebraic groups over algebraically closed fields, for $n \geq 2$.

Problem $4(\S 1)$. Suppose that $(G, \Omega)$ is a virtually definably primitive permutation group of finite Morley rank with which is not a finite cover of a definably primitive permutation group. Show that $G$ is a Chevalley group of positive characteristic, and the point stabilizer is contained in $G\left(\mathbb{F}_{q}\right)$ for some finite field $\mathbb{F}_{q}$.

Problem 5 (§1). Is there an O'Nan-Scott-Aschbacher analysis of generically 2transitive groups which are not necessarily definably primitive? Are all such groups essentially products of generically $n$-transitive primitive groups (or generically $n^{\prime}$ transitive groups, with $n^{\prime}$ not much smaller than $\left.n\right)$ ?

Problem 6 ( $(2)$. Find good bounds on $\rho$, where $\rho(r)$ is the maximum rank of a virtually definably primitive permutation group $(G, \Omega)$ of finite Morley rank, with $\operatorname{rk}(\Omega)=r$.

Problem 7 (§2). Find good bounds on $\tau$, where $\tau(r)$ is the maximum degree of generic transitivity associated to a virtually definably primitive permutation group $(G, \Omega)$ of finite Morley rank, with $\operatorname{rk}(\Omega)=r$.

Problem 8 (§5). Let $\Sigma$ be a finite group. Find lower bounds for each of the following.

(1) The minimal rank of a connected solvable group of finite Morley rank which affords a faithful representation of $\Sigma$.

(2) The minimal rank of a connected solvable group of finite Morley rank which affords a faithful representation of a central extension of $\Sigma$.

(3) The minimal rank of a connected solvable group of finite Morley rank which affords a faithful representation of a group $\hat{\Sigma}$ which covers $\Sigma$, i.e. maps homomorphically onto $\Sigma$.

Problem 9 (§6). Let $G$ be a connected group of finite Morley rank acting faithfully, definably, transitively and generically $(n+2)$-transitively on a set $\Omega$ of Morley rank $n$. Then the pair $(G, \Omega)$ is equivalent to the projective linear group $\operatorname{PGL}_{n+1}(F)$ acting on the projective space $\mathbb{P}^{n}(F)$ for some algebraically closed field $F$.

Problem $10(\S 6)$. Let $L$ be a connected $\aleph_{0}$-saturated group of finite Morley rank such that for two independent generic elements $a, b$ we have $\operatorname{rk}(d(a, b)) \leq 1$. Show that $L$ is nilpotent of bounded exponent.

Problem 11 (§6). If a simple algebraic group $L$ acts definably on a group $K$ of degenerate type, then $[K, L]$ is nilpotent.

Problem $12(\S 6)$. Let $G$ be a connected group of finite Morley rank acting faithfully, definably, and generically $t$-transitively on an abelian group $V$ of Morley rank n. Then $t \leq n$.

Problem $13(\S 6)$. Let $G$ be a connected group of finite Morley rank acting faithfully, definably, and generically n-transitively on a connected abelian group $V$ of Morley rank $n$. Then $V$ has a structure of a $n$-dimensional vector space over an 
algebraically closed field $F$ of Morley rank 1 , and $G$ is $\mathrm{GL}_{n}(F)$ in its natural action on $F^{n}$.

Problem 14 (\$6). Extend Popov's work to all characteristics and to the finite Morley rank permutation group category in which the groups are Chevalley groups, or products of Chevalley groups and tori.

Problem $15(\S 6)$. Let $G$ be a connected group of finite Morley rank acting faithfully and definably on an abelian group $V$ of Morley rank 2 . Then either $G$ is solvable, or $V$ has a structure of a 2-dimensional vector space over an algebraically closed field $F$ and $G$ is one of the groups $\mathrm{SL}_{2}(F)$ and $\mathrm{GL}_{2}(F)$ in their natural representations.

Problem 16 (§6). Let $G$ be a connected group of finite Morley rank acting faithfully, definably and irreducibly on an abelian group $V$ of Morley rank 3. Then one of the following holds:

- $V$ is of finite exponent $p>2$ and $G$ is a simple p-group.

- $V$ has a structure of a 3-dimensional vector space over an algebraically closed field $F$ and $G=Z L$ where $Z$ is a subgroup of the group of scalar matrices and $L$ is one of the groups $\mathrm{PSL}_{2}(F)$ (in its irreducible representation as a 3-dimensional orthogonal group) or $\mathrm{SL}_{3}(F)$ (in its natural action on $\left.F^{3}\right)$.

Problem 17 (§6). Let $G$ be a connected group acting definably, faithfully and irreducible on a abelian group $V$ (written additively). Assume that $G$ contains a pseudoreflection subgroup, that is, an abelian subgroup $R$ such that $V=[V, R] \oplus$ $C_{V}(R)$ and $R$ acts transitively on the set of non-trivial elements in $[V, R]$. Then $V$ has a structure of a vector space over an algebraically closed field $F$ such that $[V, R]$ is one-dimensional subspace and $R$ acts on $[V, R]$ as the multiplicative group of $F$, and $G=\mathrm{GL}(V)$.

Problem $18(\S 6)$. Let $A$ be a connected abelian group of Morley rank $n$, and let $\left(A_{i}: i \in I\right)$ be a uniformly definable family of pairwise distinct connected definable subgroups of rank $k$ in $A$. Show that $\operatorname{rk}(I) \leq\left(\begin{array}{l}n \\ k\end{array}\right)$.

\section{REFERENCES}

[1] T. Altınel, A. Borovik, and G. Cherlin, Simple Groups of Finite Morley Rank. Amer. Math. Soc. xvi+554 pp. To appear.

[2] T. Altınel and G. Cherlin, On groups of finite Morley rank of even type. J. Algebra, 264:155-185, 2003.

[3] T. Altinel and G. Cherlin, Simple $L^{*}$-groups of even type with strongly embedded subgroups. J. Algebra 272:95-127, 2004.

[4] A. Berkman, The Classical Involution Theorem for groups of finite Morley rank. J. Algebra 243:361-384, 2001.

[5] A. Borovik, J. Burdges, and G. Cherlin, Involutions in groups of finite Morley rank of degenerate type. Selecta Mathematica 13:1-22, 2007.

[6] A. Borovik, J. Burdges, and G. Cherlin, Simple groups of finite Morley rank of unipotent type. In Algebra, Logic, Set Theory. Festschrift für Ulrich Felgner zum 65. Gebutstag (B. Löwe, ed.). College Publications, 2007, pp. 47-61. ISBN 1-904987-28-1.

[7] A. Borovik and A. Nesin, Groups of Finite Morley Rank. Oxford Science Publications, The Clarendon Press, Oxford University Press, New York, 1994.

[8] S. Buechler, Essential Stability Theory. Perspectives in Mathematical Logic, Springer, 1996. ISBN 3-540-61011-1.

[9] G. Cherlin, Good tori in groups of finite Morley rank. J. Group Theory, 8:613-621, 2005. 
[10] A. Delore, Groupes simples minimaux de 2-rank de Prüfer 1. Preprint, 2005.

[11] U. Gropp, There is no sharp transitivity on $q^{6}$ when $q$ is a type of Morley rank 2. J. Symbolic Logic 57:1198-1212, 1992.

[12] M. Hall, Jr., On a theorem of Jordan. Pacific J. Mathematics 4:219-226, 1954.

[13] E. Jaligot, Generix never gives up. J. Symbolic Logic 71:599-610, 2006.

[14] H. D. Macpherson and A. Pillay, Primitive permutation groups of finite Morley rank. Proc. London Math. Soc. 70:481-504, 1995.

[15] R. Kaye and H. D. Macpherson, Automorphisms of First-Order Structures. Oxford Science Publications, The Clarendon Press, Oxford University, 1994. ISBN 0-19853468-X.

[16] U. Meierfrankenfeld, A characterization of the natural module for classical groups. Preprint.

[17] B. Poizat, Groupes Stables. Nur Al-Mantiq Wal-Ma'rifah, Villeurbanne, France, 1987; English translation 2001, AMS

[18] B. Poizat, Modestes remarques a propos d'une conséquence inattendue d'un résultat surprenant de Monsieur Frank Olaf Wagner. J. Symbolic Logic 66:1637-1646, 2001

[19] V. Popov, Generically multiply transitive algebraic group actions. Preprint, 2005.

[20] F. Timmesfeld, Abstract Root Subgroups and Simple Groups of Lie-Type. Monographs in Mathematics 95, Birkhäuser, Basel, 2001, xiii+389 pp.

[21] F. Wagner, Fields of finite Morley rank. J. Symbolic Logic, 66:703-706, 2001.

E-mail address: borovik@manchester.ac.uk 